\title{
Strong Local Consistency Algorithms for Table Constraints *
}

\author{
Anastasia Paparrizou $^{\dagger} \quad$ Kostas Stergiou
}

\begin{abstract}
Table constraints are important in constraint programming as they are present in many real problems from areas such as configuration and databases. As a result, numerous specialized algorithms that achieve generalized arc consistency (GAC) on table constraints have been proposed. Since these algorithms achieve GAC, they operate on one constraint at a time. In this paper we propose new filtering algorithms for positive table constraints that achieve stronger local consistency properties than GAC by exploiting intersections between constraints. The first algorithm, called maxRPWC+, is a domain filtering algorithm that is based on the local consistency maxRPWC and extends the GAC algorithm of Lecoutre and Szymanek [23]. The second algorithm extends the state-of-the-art STR-based algorithms to stronger relation filtering consistencies, i.e., consistencies that can remove tuples from constraints' relations. Experimental results from benchmark problems demonstrate that the proposed algorithms are quite competitive with standard GAC algorithms like STR2 in some classes of problems with intersecting table constraints, being orders of magnitude faster in some cases.
\end{abstract}

\section{Introduction}

Table constraints, i.e., constraints given in extension, are ubiquitous in constraint programming (CP). First, they naturally arise in many real applications from areas such as configuration and databases. And second, they are a useful modeling tool that can be called upon to, for instance, easily capture preferences [15]. Given their importance in $\mathrm{CP}$, it is not surprising that table constraints are among the most widely studied constraints and as a result numerous specialized algorithms that achieve generalized arc consistency (GAC) on them have been proposed.

GAC algorithms for positive table constraints, i.e., constraints defined by sets of allowed tuples, have received the bulk of the attention. Such algorithms utilize a number of different techniques to speed-up the check for generalized arc consistency. For example, some of them build upon GAC-schema [4] by interleaving the exploration of allowed and valid tuples using either intricate data structures [26] or binary search [23]. Other methods compile the tables into efficient data structures that allow for

* Some results included in this paper first appeared in [31].

${ }^{\dagger}$ This author has been funded by the EU project ICON (FP7-284715). 
faster support search (e.g., [11] and [9]). Simple Tabular Reduction (STR) [34] and its refinements $[18,22]$ maintain dynamically the support tables by removing invalid tuples from them during search. A recent approach holds information about removed values in the propagation queue and utilizes it to speed up support search [29].

Given that GAC is a property defined on individual constraints, algorithms for GAC operate on one constraint at a time trying to filter infeasible values from the variables of the constraint. A different line of research has investigated stronger consistencies and algorithms to enforce them. Some of them are domain filtering, meaning that they only prune values from the domains of variables, e.g., see [10,7], whereas a few other ones are higher-order (or relation filtering), e.g., see [12, 13, 35, 16, 20], indicating that inconsistent tuples of values (nogoods of size 2 or more) can be identified. In contrast to GAC algorithms, the proposed algorithms to enforce these stronger consistencies are able to consider several constraints simultaneously. For example, pairwise consistency (PWC) [12] is a relation filtering consistency that considers intersections between pairs of constraints.

Recently there has been renewed interest for strong domain or relation filtering local consistencies as new ones have been proposed or/and efficient algorithms for existing ones have been devised [19, 7, 16, 37]. One of the most promising such consistencies is Max Restricted Pairwise Consistency (maxRPWC) [7], which is local consistency that is based PWC but can only make value deletions. In practice, strong consistencies are mainly applicable on constraints that are extensionally defined since intensionally defined constraints usually have specific semantics and are provided with efficient specialized filtering algorithms. However, a significant shortcoming of existing works on maxRPWC and other strong local consistencies is that the proposed algorithms for them are generic. That is, like earlier GAC algorithms such as GAC2001/3.1 [6], they are designed to operate on both intensional and extensional constraints, failing to recognize that strong consistencies are predominantly applicable on extensional constraints and should thus focus on such constraints.

Despite the wealth of research on strong consistencies, they have not been widely adopted by CP solvers. State-of-the art solvers such as Gecode, Abscon, Choco, Minion, etc. predominantly apply GAC, and lesser forms of consistency such as bounds consistency, when propagating constraints. Regarding table constraints, CP solvers typically offer one or more of the above mentioned GAC methods for propagation.

In this paper we propose filtering algorithms that achieve stronger consistency properties than GAC and have been specifically designed for table constraints. We contribute to both directions of domain and relation filtering methods by extending existing GAC algorithms for table constraints. The proposed methods are a step towards the efficient handling of intersecting table constraints and also provide specialization of strong local consistencies to extensional constraints that can be useful in practice.

The first algorithm, called maxRPWC+, extends the GAC algorithm for table constraints of Lecoutre and Szymanek [23] and specializes the generic maxRWPC algorithm maxRPWC1 [7]. The proposed domain filtering algorithm incorporates several techniques that help alleviate redundancies (i.e., redundant constraint checks and other operations on data structures) displayed by existing maxRPWC algorithms. We also describe a variant of maxRPWC+ which is more efficient when applied during search due to the lighter use of data structures. 
The second algorithm, called HOSTR $\star$, extends the state-of-the-art GAC algorithm STR to a higher-order local consistency that can delete tuples from constraint relations as well as values from domains. HOSTR $\star$ is actually a family of algorithms that combines the operation of STR (or a refinement of STR such as STR2) when establishing GAC on individual constraints and the operation of maxRPWC+ when trying to ex-

tend GAC supports to intersecting constraints. We describe several instantiations of HOSTR* which differ in their implementation details.

We theoretically study the pruning power of the proposed algorithms and place them in a partial hierarchy which includes GAC, maxRPWC, and full pairwise consistency (FPWC), which is pairwise consistency followed by GAC. We show that the level of local consistency achieved by HOSTR $\star$ is incomparable to that achieved by maxRPWC+ and to maxRPWC, but weaker than FPWC. Interestingly, a simple variant of HOSTR $\star$ achieves FPWC, albeit with a high cost. maxRPWC+ achieves a consistency that is incomparable to maxRPWC but still stronger than GAC.

Experimental results from benchmark problems used in the evaluation of filtering algorithms for table constraints demonstrate that the proposed algorithms are considerably faster than the generic maxRPWC1 algorithm in classes of problems with large tables. Also, the best among the proposed algorithms outperforms the state-of-the-art GAC algorithm STR2 on some problem classes with intersecting table constraints, being orders of magnitude faster in many cases. However, it is also outperformed by STR2 on other problem classes with the differences being quite large in some cases.

We also compare our approaches to the state-of-the-art Abscon solver which is a solver with a more optimized implementation than our own. Results demonstrate that Abscon is faster on most problem classes, but since our methods often cut down the size of the search tree considerably, there are quite a few cases where this is reflected on important CPU time gains.

Finally, comparing our methods to eSTR2, a relevant later introduced algorithm [24], we observe that although eSTR2 is faster on most problems, its high memory requirements mean that its use is infeasible on certain instances. In contrast, our algorithms do not suffer from the drawback of high memory consumption and can easily handle the instances where eSTR2 fails.

The rest of this paper is structured as follows. Section 2 gives the necessary background. Section 3 presents algorithm maxRPWC+ and a variant of this algorithm that is more efficient when used during search. Section 4 extends STR-based algorithms to achieve stronger consistencies than GAC. In Section 5 we give experimental results. Finally, in Sections 6 and 7 we discuss related work and we conclude.

\section{Background}

A Constraint Satisfaction Problem $(\mathrm{CSP})$ is defined as a tuple $(\mathcal{X}, \mathcal{D}, \mathcal{C})$ where:

- $\mathcal{X}=\left\{x_{1}, \ldots, x_{n}\right\}$ is a set of $n$ variables.

- $\mathcal{D}=\left\{D\left(x_{1}\right), \ldots, D\left(x_{n}\right)\right\}$ is a set of finite domains, one for each variable, with maximum cardinality $d$. 
- $\mathcal{C}=\left\{c_{1}, \ldots, c_{e}\right\}$ is a set of $e$ constraints; with $k$ denoting the maximum arity of the constraints.

Constraints can be defined either extensionally by listing the allowed (or disallowed) combinations of values or intensionally through a predicate or a function. A positive table constraint $c_{i}$ is a constraint given in extension and defined by a set of allowed tuples. A tuple is called allowed iff it satisfies $c_{i}$. Each table constraint $c_{i}$ is a pair $\left(\operatorname{scp}\left(c_{i}\right), \operatorname{rel}\left[c_{i}\right]\right)$, where $\operatorname{scp}\left(c_{i}\right)=\left\{x_{i_{1}}, \ldots, x_{i_{r}}\right\}$ is an ordered subset of $\mathcal{X}$ referred to as the constraint scope, and $\operatorname{rel}\left[c_{i}\right]$ is a subset of the Cartesian product $D\left(x_{i_{1}}\right) \times \ldots \times D\left(x_{i_{r}}\right)$ that specifies the allowed combinations of values (known also as $c_{i}$ 's relation) for the variables in $\operatorname{scp}\left(c_{i}\right)$. For each table constraint $c_{i} \in \mathcal{C}$, we have $t_{c_{i}}=\left|\operatorname{rel}\left[c_{i}\right]\right|$ and $t=\operatorname{maximum}\left(t_{c_{i}}\right)$.

An assigment of a value $a_{i}$ to a variable $x_{i}$ is denoted by $\left(x_{i}, a_{i}\right)$. A tuple $\tau \in$ $\operatorname{rel}\left[c_{i}\right]$ is an ordered list of value to variable assignments $\left(\left(x_{1}, a_{1}\right), \ldots,\left(x_{m}, a_{m}\right)\right)$ s.t. $a_{j} \in D\left(x_{j}\right), j=1, \ldots, m$. Given a (table) constraint $c_{i}$, and a tuple $\tau \in \operatorname{rel}\left[c_{i}\right]$, we denote by $\tau[x]$ the projection of $\tau$ on a variable $x \in \operatorname{scp}\left(c_{i}\right)$ and by $\tau[X]$ the projection of $\tau$ on any subset $X \subseteq \operatorname{scp}\left(c_{i}\right)$ of variables; $\tau[X]$ is called a subtuple of $\tau$. A tuple is valid iff none of the values in the tuple has been removed from the variable domains of the corresponding variable. For any constraint $c_{i}$ we denote by $\top$ (resp. $\perp$ ) a dummy tuple s.t. $\tau\left\langle\top\right.$ (resp. $\tau>\perp$ ) for any tuple $\tau \in \operatorname{rel}\left[c_{i}\right]$. We assume that for any table constraint its tuples are stored in lexicographical order.

Given two constraints $c_{i}$ and $c_{j}$, if $\left|s c p\left(c_{i}\right) \cap s c p\left(c_{j}\right)\right|>1$ we say that the constraints intersect non trivially. We denote by $f_{\min }$ the minimum number of variables that are common to any two constraints that intersect on more than one variable ${ }^{1}$. Therefore, $f_{\min }$ is at least two.

The most commonly used local consistency is generalized arc consistency (GAC) or domain consistency. A value $a_{i} \in D\left(x_{i}\right)$ is GAC iff for every constraint $c$, s.t. $x_{i} \in \operatorname{scp}(c)$, there exists a valid tuple $\tau \in \operatorname{rel}[c]$ s.t. $\tau\left[x_{i}\right]=a_{i}[30,28]$. In this case $\tau$ is a GAC-support of $a_{i}$ on $c$. A variable is GAC iff all values in its domain are GAC. A problem is GAC iff there is no empty domain in $\mathcal{D}$ and all variables are GAC.

Strong Local Consistencies Several alternative local consistencies have been proposed, with domain filtering consistencies being especially interesting since they only remove values from domains and thus do not alter the structure of the constraint (hyper)graph or the constraints' relations. Examples of such consistencies for nonbinary constraints include SAC [10], RPWC and maxRPWC [7], and rPIC [33]. Some of the consistencies, are directly defined on both binary and non-binary constraints (i.e., SAC for binary and non-binary constraints). Others, like maxRPWC, are defined on non-binary constraints but are inspired by relevant consistencies for binary constraints. A theoretical and experimental evaluation demonstrated that maxRPWC is a promising alternative to GAC [7].

A value $a \in D\left(x_{i}\right)$ is max Restricted Pairwise Consistent (maxRPWC) iff $\forall c_{j} \in C$, where $x_{i} \in \operatorname{scp}\left(c_{j}\right), a$ has a GAC-support $\tau \in \operatorname{rel}\left[c_{j}\right]$ s.t. $\forall c_{l} \in C\left(c_{l} \neq c_{j}\right)$, s.t. $s c p\left(c_{j}\right) \cap s c p\left(c_{l}\right) \neq \emptyset, \exists \tau^{\prime} \in \operatorname{rel}\left[c_{l}\right]$, s.t. $\tau\left[\operatorname{scp}\left(c_{j}\right) \cap s c p\left(c_{l}\right)\right]=\tau^{\prime}\left[\operatorname{scp}\left(c_{j}\right) \cap s c p\left(c_{l}\right)\right]$

\footnotetext{
${ }^{1}$ On constraints that intersect on one variable maxRPWC is equivalent to GAC [7].
} 
and $\tau^{\prime}$ is valid. In this case we say that $\tau^{\prime}$ is a PW-support of $\tau$ and $\tau$ is a maxRPWCsupport of $a$. A variable is maxRPWC iff all values in its domain are maxRPWC. A problem is maxRPWC iff there is no empty domain in $\mathcal{D}$ and all variables are maxRPWC.

GAC and maxRPWC are domain filtering (or first-order) consistencies, meaning that they can only identify inconsistent values, thereby filtering variable domains. Relation filtering (or higher-order) consistencies allow us to identify inconsistent tuples of values in constraints' relations. This is the case of pairwise consistency (PWC) defined as follows (for positive table constraints). A tuple $\tau_{i}$ in the table of a constraint $c_{i}$ is PWC iff $\forall c_{j} \in \mathcal{C}, \exists \tau_{j}$ in $\operatorname{rel}\left[c_{j}\right]$ which is a PW-support of $\tau_{i}$. A problem is PWC iff every tuple of every constraint in the problem is PWC. We will also say that a problem is FPWC iff it is both PWC and GAC.

Following Debruyne and Bessiere [10], we call a local consistency $A$ stronger than $B$ iff in any problem in which $A$ holds then $B$ holds, and strictly stronger iff it is stronger and there is at least one problem in which $B$ holds but $A$ does not. Accordingly, $A$ is incomparable to $B$ iff none is stronger than the other.

Three algorithms for achieving maxRPWC have been proposed [7]. The first one, maxRPWC1, has $\mathrm{O}\left(e^{2} k^{2} d^{p}\right)$ worst-case time complexity and $\mathrm{O}(e k d)$ space complexity, where $p$ is the maximum number of variables involved in two constraints that share at least two variables. The second one has $\mathrm{O}\left(e^{2} k d^{k}\right)$ time complexity but its space complexity is exponential in $p$, and this can be prohibitive. The third one has the same time complexity as maxRPWC1 but $\mathrm{O}\left(e^{2} k d\right)$ space complexity. Although maxRPWC1 is less sophisticated than the other two, its performance when maintained during search is on average better than theirs because it uses lighter data structures. All these algorithms are generic in the sense that they do not consider any specific semantics that the constraints may have.

Recently, an extension of STR-based algorithms, called eSTR, that achieves full pairwise consistency was proposed [24]. This algorithm verifies the existence of a PW-support, in constant time, through the use of counters that store the number of occurrences of specific combinations of values in constraint intersections. As a result, the worst-case time complexity of one call to its basic filtering procedure (i.e., the revision of a constraint's table) is quite close to that of STR algorithms. Precisely, it is $\mathrm{O}(k d+\max (k, g) t)$, where $g$ is the maximum number of non-trivial intersections that a constraint may have, while its worst-case space complexity of handling one constraint is $\mathrm{O}(n+\max (k, g) t)$. In practice, the space requirements, even for just one constraint, can be quite high because eSTR requires $\mathrm{O}(t)$ additional space for each intersecting constraint. A weaker version of eSTR, denoted by eSTR ${ }^{w}$, was also proposed. This achieves a consistency level between GAC and FPWC and is more efficient in practice. Experiments demonstrated that this method can significantly outperform STR2 in many problem classes, but it can become prohibitive when many and/or large intersections occur, due to its high space requirements. 


\section{Algorithm maxRPWC+}

In this section we will first describe an algorithm that is based on maxRPWC and is specialized for application on table constraints, and then present an efficient variant of it that makes a lighter use of its data structures. The presented algorithm, called maxRPWC+, builds upon the generic maxRPWC algorithm maxRPWC1 and a GAC algorithm that is specific to table constraints (called GAC-va hereafter) [23]. maxRPWC+ not only specializes maxRPWC to table constraints but also introduces several techniques that help eliminate redundancies displayed by existing algorithms, such as unnecessary constraint checks and other operations on data structures. As in GAC-Va, the main idea behind maxRPWC+ is to interleave support and validity checks.

The approach of GAC-va involves visiting both lists of valid and allowed tuples in an alternating fashion when looking for a support (i.e., a tuple that is both allowed and valid). Its principle is to avoid considering irrelevant tuples by jumping over sequences of valid tuples containing no allowed tuple and over sequences of allowed tuples containing no valid tuple. This is made possible because of the lexicographic ordering of tuples. The core operation of GAC-va, which is also exploited by our algorithm, is the construction of a valid tuple that is verified for being a GAC-support by searching for it in the list of allowed tuples using binary search. If it is not found, then the smallest allowed tuple that is greater than the aforementioned valid one is considered and its validity is checked. If it is not valid, then next valid tuple is constructed and so on.

Algorithm maxRPWC+ uses the following data structures:

- For each constraint $c$ and each value $a_{i} \in D\left(x_{i}\right)$, where $x_{i} \in \operatorname{scp}(c)$, allowed $(c$, $\left.x_{i}, a_{i}\right)$ is the list of allowed tuples in $c$ that include the assignment $\left(x_{i}, a_{i}\right)$.

- For each constraint $c$ and each value $a_{i} \in D\left(x_{i}\right)$, where $x_{i} \in \operatorname{scp}(c)$, Last $_{c, x_{i}, a_{i}}$ gives the most recently discovered (and thus lexicographically smallest) maxRPWCsupport of $a_{i}$ in $c$. The same data structure is used by maxRPWC1 but it is exploited in a less sophisticated way as will be explained in Section 3.1.

Before going into the details of the algorithm we describe a simple modification that can be incorporated into any maxRPWC algorithm to boost its performance.

Restricted maxRPWC From the definition of maxRPWC we can see that the value deletions from some $D\left(x_{i}\right)$ may trigger the deletion of a value $b \in D\left(x_{j}\right)$ in two cases:

1. $b$ may no longer be maxRPWC because its current maxRPWC-support in some constraint $c$ is no longer valid and it was the last such support in $c$. We call this case maxRPWC-support loss.

2. The last maxRPWC-support of $b$ in some constraint $c$ may have lost its last PWsupport in another constraint $c^{\prime}$ intersecting with $c$. We call this case $P W$-support loss.

Although detecting PW-support loss is necessary for an algorithm to achieve maxRPWC, our experiments have shown that the pruning it achieves rarely justifies its cost. Hence, maxRPWC+ applies maxRPWC in a restricted way by only detecting 
maxRPWC-support loss. However, the resulting method is still strictly stronger than GAC. This is clear if we consider that the "stronger" relationship is immediately derived by the definitions. Now consider a problem with constraints alldiff( $\left.x_{1}, x_{2}, x_{3}\right)$ and $x_{1}=x_{2}$, and domains $\{0,1,2\}$ for all variables. This problem is GAC but the application of restricted maxRPWC will detect its inconsistency. Although a restricted version of maxRPWC is stronger than GAC, it obviously only achieves an approximation of maxRPWC. A similar approximation of the related binary local consistency maxRPC has also been shown to be efficient compared to full maxRPC [36, 1].

\subsection{Algorithm description}

Given a table constraint $c_{i}$, we now describe how algorithm maxRPWC+ can be used to filter the domain of any variable $x_{j} \in \operatorname{scp}\left(c_{i}\right)$. We assume that the domain of some variable in $\operatorname{scp}\left(c_{i}\right)$ (different from $x_{j}$ ) has been modified and as a result the propagation engine will revise all other variables in $\operatorname{scp}\left(c_{i}\right)$. Initially, Function 1 is called.

For each value $a_{j} \in D\left(x_{j}\right)$ Function 1 first searches for a GAC-support. This is done by calling function seekSupport-va which is an adaptation of Algorithm 12 of GAC-va [23]. This function makes an additional first check to verify if Last $_{c_{i}, x_{j}, a_{j}}$, which is the most recently found maxRPWC-support, and thus also GAC-support, is still valid. Note that the search starts from the first valid tuple. If $\operatorname{Last}_{c_{i}, x_{j}, a_{j}}$ is valid, $\tau=$ Last $_{c_{i}, x_{j}, a_{j}}$ is returned, else the valid and allowed tuples of $c_{i}$ are visited in an alternating fashion. This is done by applying a dichotomic search in the list allowed $\left(c_{i}, x_{j}, a_{j}\right)$ to locate the lexicographically smallest valid and allowed tuple $\tau$ of $c_{i}$, such that $\tau>$ Last $_{c_{i}, x_{j}, a_{j}}$ and $\tau\left[x_{j}\right]=a_{j}$. More precisely, $\tau$ can be either a valid tuple found in the list of allowed tuples of $c_{i}$ or $T$, in case of validity or support check failure. If such a tuple $\tau$ is found, we then check it for PW consistency through Function isPWconsistent+ (Function 2). If $a_{j}$ does not have a GAC-support (i.e., seekSupport-va returns $T$ ) or none of its GAC-supports is a PW-support, then it will be removed from $D\left(x_{j}\right)$.

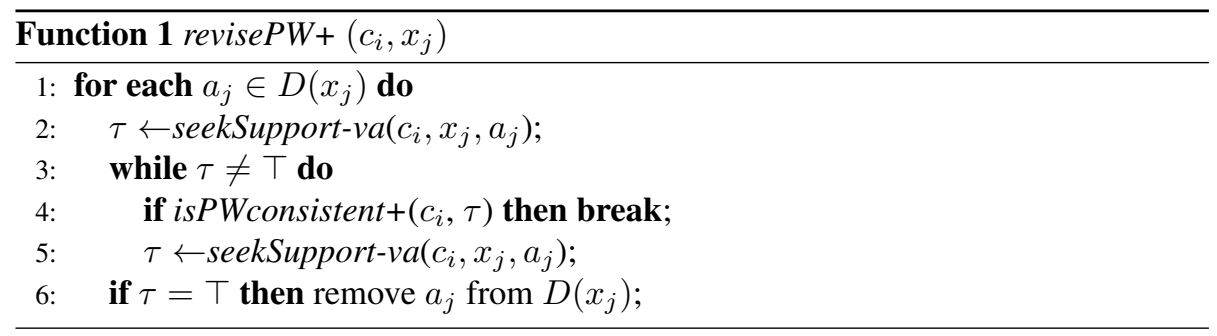

The process of checking if a tuple $\tau$ of a constraint $c_{i}$ is PW consistent involves iterating over each constraint $c_{k}$ that intersects with $c_{i}$ on at least two variables and searching for a PW-support for $\tau$ (Function 2 line 1). For each such constraint $c_{k}$ maxRPWC+ first tries to quickly verify if a PW-support for $\tau$ exists by exploiting the Last data structure as we now explain. 


\subsubsection{Fast check for PW-support}

For each variable $x_{k}$ belonging to the intersection of $c_{i}$ and $c_{k}$, we check if $\tau^{\prime}=$ Last $_{c_{k}, x_{k}, \tau\left[x_{k}\right]}$ is valid and if it includes the same values for the rest of the variables in the intersection as $\tau$ (line 6 in Function 2). Function isValid simply checks if all values in the tuple are still in the domains of the corresponding variables. If these conditions hold for some variable $x_{k}$ in the intersection then $\tau$ is PW-supported by $\tau^{\prime}$. Hence, we move on to the next constraint intersecting $c_{i}$.

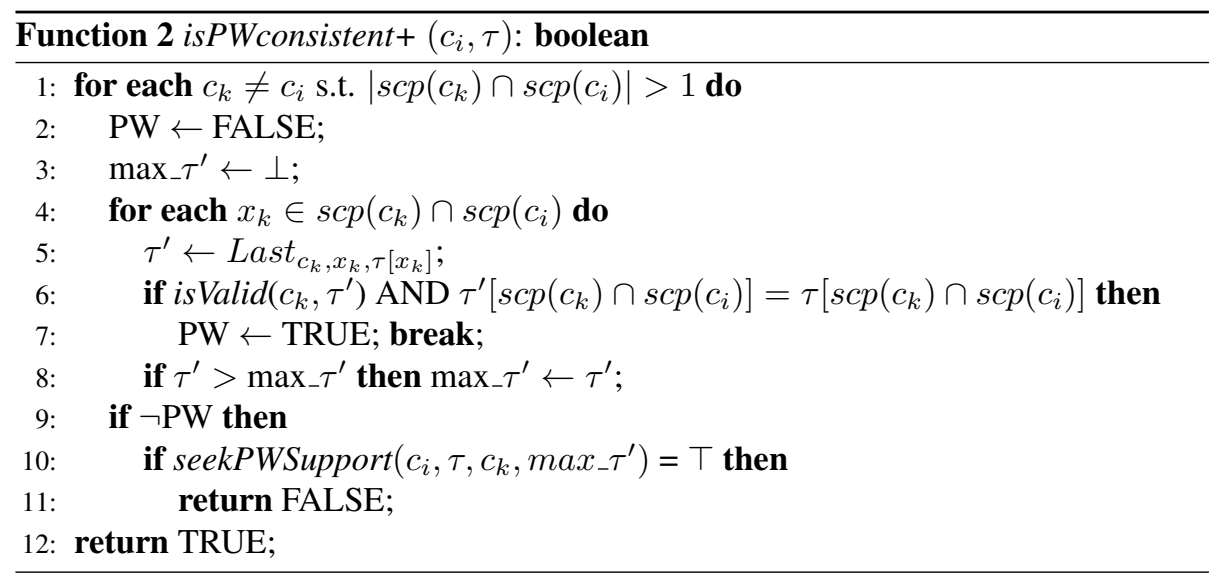

Else, we find $\max \_\tau^{\prime}$ the lexicographically greatest $\operatorname{Last}_{c_{k}, x_{k}, \tau\left[x_{k}\right]}$ among the variables that belong to the intersection of $c_{i}$ and $c_{k}$ and we search for a new PW-support in Function seekPWSupport (line 10). In case seekPWSupport returns $T$ for some $c_{k}$ then isPWconsistent+ returns FALSE and a new GAC-support must be found and checked for PW consistency.

\subsubsection{Fast check for lack of PW-support}

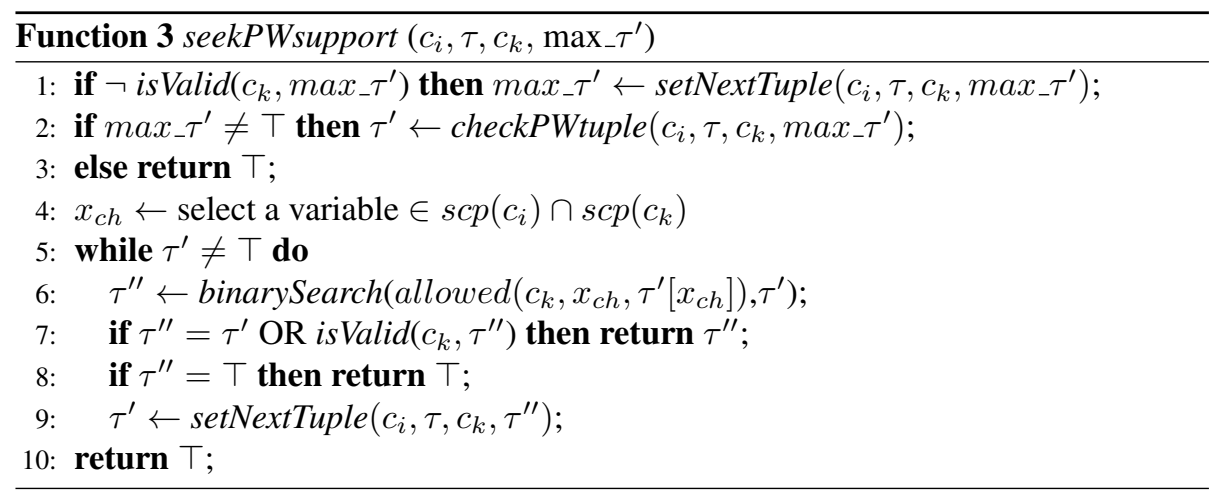

Function 3 seeks a PW-support for $\tau$ in $\operatorname{rel}\left[c_{k}\right]$. Before commencing with this search, it performs a fast check aiming at detecting a possible inconsistency (and thus 
avoiding the search). In a few words, this check can sometimes establish that there cannot exist a PW-support for $\tau$. This is accomplished by exploiting the lexicographical ordering of the tuples in the constraints' relations.

In detail, the validity of $\max _{-} \tau^{\prime}$ is first checked in line 1. If is Valid returns FALSE, then function setNextTuple is called to find the lexicographically smallest valid tuple in $c_{k}$ that is greater than $\max _{-} \tau^{\prime}$ and is such that $\max _{-} \tau^{\prime}\left[\operatorname{scp}\left(c_{k}\right) \cap \operatorname{scp}\left(c_{i}\right)\right]=\tau\left[\operatorname{scp}\left(c_{k}\right) \cap\right.$ $\operatorname{scp}\left(c_{i}\right)$ ]. If no such tuple exists, setNextTuple returns $\top$, and the search terminates since no PW-support for $\tau$ exists in $c_{k}$. If a tuple $\max _{-} \tau^{\prime}$ is located then Function checkPWtuple is called to essentially perform a lexicographical comparison between $\max _{-} \tau^{\prime}$ and $\tau$ taking into account the intersection of the two constraints (line 2 in Function 3). According to the result we may conclude that there can be no PW-support of $\tau$ in $c_{k}$ and thus Function 3 will return $T$. Consequently, lines 2-3 of Function 3 perform the fast check for lack of PW-support.

The addition of this simple check enables maxRPWC+ to perform extra pruning compared to a typical maxRPWC algorithm. Before explaining how checkPWtuple works, we demonstrate this with an example.

Example 1 Consider a problem that includes two constraints $c_{1}$ and $c_{2}$ with $\operatorname{scp}\left(c_{1}\right)=$ $\left\{x_{1}, x_{2}, x_{3}, x_{4}\right\}$ and $\operatorname{scp}\left(c_{2}\right)=\left\{x_{3}, x_{4}, x_{5}, x_{6}\right\}$. Assume that the GAC-support $\tau=$ $(0,2,2,1)$ has been located for value 0 of $x_{1}$ and that there exists a valid PW-support for $\tau$ in $c_{2}$ (e.g., $\{2,1,2,2\}$ ). Also, assume that Last $_{c_{2}, x_{3}, 2}$ and Last $_{c_{2}, x_{4}, 1}$ are tuples $\tau^{\prime}=(2,2,0,1)$ and $\tau^{\prime \prime}=(1,1,2,3)$, meaning that $\max _{-} \tau^{\prime}=\tau^{\prime}$. Since $\tau$ has a PW-support, a maxRPWC algorithm will discover this and will continue to check the next constraint intersecting $c_{1}$. However, since $\tau^{\prime}\left[x_{4}\right]$ is greater than $\tau\left[x_{4}\right]$, it is clear that there is no PW consistent tuple in $c_{2}$ that includes values 2 and 1 for $x_{3}$ and $x_{4}$ respectively. If we assume that $\tau$ is the last GAC-support of $\left(x_{1}, 0\right)$ then maxRPWC+ will detect this and will delete 0 from $D\left(x_{1}\right)$, while a maxRPWC algorithm will not.

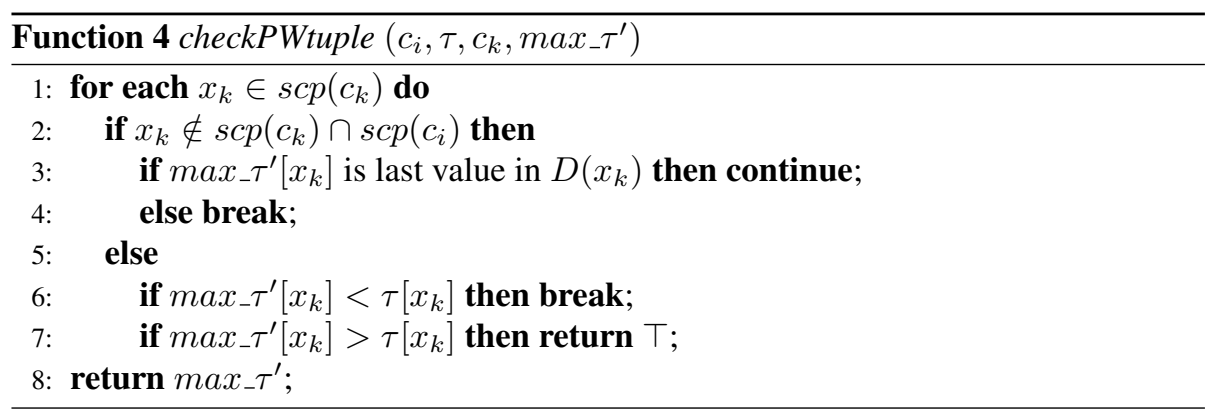

Function checkPWtuple (Function 4) checks if there can exist a tuple greater or equal to $\max _{-} \tau^{\prime}$ that has the same values for the variables of the intersection as $\tau$. Crucially, this check is done in linear time as follows: Assuming $\max _{-} \tau^{\prime}=\left\langle\left(x_{1}, a_{1}\right), \ldots\right.$, $\left.\left(x_{m}, a_{m}\right)\right\rangle$ then this tuple is scanned from left to right. If the currently examined variable $x_{k}$ belongs to $\operatorname{scp}\left(c_{k}\right) \cap \operatorname{scp}\left(c_{i}\right)$ and $a_{k}>\tau\left[x_{k}\right]$, where $a_{k}$ is the value of $x_{k}$ in $\max _{-} \tau^{\prime}$, then we conclude that there can be no PW-support for $\tau$ in $c_{k}$ (line 7). If $x_{k}$ does not belong to $\operatorname{scp}\left(c_{k}\right) \cap s c p\left(c_{i}\right)$ then if the value it takes in $\max _{-} \tau^{\prime}$ is the last value 
in its domain, we continue searching (line 3). Otherwise, the scan is stopped because there may exist a tuple larger or equal to $\max _{-} \tau^{\prime}$ that potentially is a PW-support of $\tau$. However, $\max _{-} \tau^{\prime}$ can still be used to avoid searching for a PW-support from scratch. Hence it is returned to seekPWsupport.

The soundness of the described process is guaranteed by the assumption that tuples in relations are stored in lexicographical order, which is typically the case. Given this assumption, it is certain that if a tuple $\tau$ which we try to extend to a PW-support is lexicographically smaller than $\max _{-} \tau^{\prime}$, with respect to the values of the shared variables, then there can be no PW-support for $\tau$. Otherwise, the lexicographical order would be violated.

\subsubsection{Searching for PW-support}

In case no inconsistency is detected through the fast check, then the search for a PWsupport for $\tau$ begins, starting with the tuple $\tau^{\prime}$ returned from checkPWtuple. We first check if $\tau^{\prime}$ is an allowed tuple using binary search in a similar way to GAC-va. However, since there are more than one variables in the intersection of $c_{i}$ and $c_{k}$, the question is which list of allowed tuples to consider when searching. Let us assume that the search will be performed on the list allowed $\left(c_{k}, x_{c h}, \tau^{\prime}\left[x_{c h}\right]\right)$ of variable $x_{c h}$. After describing the process, we will discuss possible criteria for choosing this variable.

Binary search will either return $\tau^{\prime}$ if it is indeed allowed, or the lexicographically smallest allowed tuple $\tau^{\prime \prime}$ that is greater than $\tau^{\prime}$, or $\top$ if no such tuple exists. In the first case a PW-support for $\tau$ has been located and it is returned. In the third case, no PWsupport exists. In the second case, we check if $\tau^{\prime \prime}$ is valid, by using function is Valid and if so, then it constitutes a PW-support for $\tau$. Otherwise, function setNextTuple is called taking $\tau^{\prime \prime}$ and returning the smallest valid tuple for $\operatorname{scp}\left(c_{k}\right)$ that is lexicographically greater than $\tau^{\prime \prime}$, such that $\tau^{\prime}\left[\operatorname{scp}\left(c_{k}\right) \cap \operatorname{scp}\left(c_{i}\right)\right]=\tau\left[\operatorname{scp}\left(c_{k}\right) \cap \operatorname{scp}\left(c_{i}\right)\right]$ (line 9). If setNextTuple returns $\top$ the search terminates, otherwise, we continue to check if the returned tuple is allowed as explained above, and so on.

\subsubsection{Selecting the list of allowed tuples}

Since there are $\left|s c p\left(c_{k}\right) \cap s c p\left(c_{i}\right)\right|$ variables in the intersection of $c_{i}$ and $c_{k}$, there is the same number of choices for the list of allowed tuples to be searched. Obviously, the size of the lists is a factor that needs to be taken into account. The selection of $x_{c h}$ (line 4 of Function 3) can be based on any of the following (and possibly other) criteria:

1. Select the variable $x_{c h}$ having minimum size of allowed $\left(c_{k}, x_{c h}, \tau^{\prime}\left[x_{c h}\right]\right)$.

2. Select the variable $x_{c h}$ having the minimum number of tuples between $\tau^{\prime}$ and $T$ in $\operatorname{allowed}\left(c_{k}, x_{c h}, \tau^{\prime}\left[x_{c h}\right]\right)$.

3. Select the leftmost variable in $s c p\left(c_{k}\right) \cap \operatorname{scp}\left(c_{i}\right)$.

4. Select the rightmost variable in $s c p\left(c_{k}\right) \cap \operatorname{scp}\left(c_{i}\right)$.

The first heuristic considers a static measure of the size of the lists. The second considers a more dynamic and accurate measure. In the experiments, presented below, 
we have used the fourth selection criterion. Although this seems simplistic, as Example 2 demonstrates, there are potentially significant benefits in choosing the rightmost variable.

Example 2 Consider a constraint $c_{k}$ on variables $x_{1}, \ldots, x_{4}$ with domains $D\left(x_{1}\right)=$ $D\left(x_{4}\right)=\{0, \ldots, 9\}$ and $D\left(x_{2}\right)=D\left(x_{3}\right)=\{0,1\}$. Assume that we are seeking a PW-support for tuple $\tau$ of constraint $c_{i}$ in $c_{k}$. Also, $\operatorname{scp}\left(c_{k}\right) \cap \operatorname{scp}\left(c_{i}\right)=\left\{x_{1}, x_{4}\right\}$, $\tau\left[x_{1}\right]=1, \tau\left[x_{4}\right]=0$, and $\left|\operatorname{allowed}\left(c_{k}, x_{1}, 1\right)\right|=\left|\operatorname{allowed}\left(c_{k}, x_{4}, 0\right)\right|$. Figure 1 (partly) shows the lists allowed $\left(c_{k}, x_{1}, 1\right)$ and allowed $\left(c_{k}, x_{4}, 0\right)$. If we choose to search for a PW-support in allowed $\left(c_{k}, x_{1}, 1\right)$ then in the worst case binary search will traverse the whole list since tuples with value 0 for $x_{4}$ are scattered throughout the list. In contrast, if we choose allowed $\left(c_{k}, x_{4}, 0\right)$ then search can focus in the highlighted part of the list since tuples with value 1 for $x_{1}$ are grouped together.

\begin{tabular}{cccc}
$\mathrm{x} 1$ & $\mathrm{x} 2$ & $\mathrm{x3}$ & $\mathrm{x4}$ \\
\hline 1 & $\mathbf{0}$ & $\mathbf{0}$ & $\mathbf{0}$ \\
$\ldots .$. & $\ldots$ & $\ldots$ & $\ldots$ \\
1 & 0 & 0 & 9 \\
1 & 1 & 0 & 0 \\
$\ldots$. & $\ldots$ & $\ldots$ & $\ldots$ \\
1 & 1 & 0 & 9 \\
$\cdots$. & $\ldots$ & $\ldots$ & $\ldots$ \\
1 & 1 & 1 & 9
\end{tabular}

\begin{tabular}{|c|c|c|c|}
\hline x1 & $\mathbf{x} 2$ & x3 & x4 \\
\hline 0 & 0 & 0 & 0 \\
\hline$\ldots$ & $\ldots$ & $\ldots$ & $\cdots$ \\
\hline 0 & 1 & 1 & $\mathbf{0}$ \\
\hline 1 & 0 & 0 & 0 \\
\hline$\ldots$ & $\cdots$ & $\ldots$ & $\ldots$ \\
\hline 1 & 1 & 1 & 0 \\
\hline$\ldots$ & ... & $\ldots$ & $\cdots$ \\
\hline 9 & 1 & 1 & 0 \\
\hline
\end{tabular}

Figure 1: allowed $\left(c_{k}, x_{1}, 1\right)$ and allowed $\left(c_{k}, x_{4}, 0\right)$.

\subsection{Theoretical Results}

We now analyze the worst-case complexity of the revisePW+ function of maxRPWC+. The symbols $M, N, S$ are explained in the proof.

Proposition 1 The worst-case time complexity of reviseP $W+\left(c_{i}, x_{j}\right)$ is $\mathrm{O}($ d.e.N.M $(d+$ $k . \log (S)))$.

Proof: Let us first consider the complexities of the individual functions called by seekPWsupport. The cost of setNextTuple to construct a valid tuple for the variables that do not belong to the intersection is $\mathrm{O}\left(d+\left(k-f_{\text {min }}\right)\right)$. The cost of checkPWtuple is linear, since it requires at most $\mathrm{O}(k)$ checks to determine if any of $x_{k} \in c_{k}$ is inconsistent with $\tau\left[x_{k}\right]$. The worst-case time complexity of binarySearch is $\mathrm{O}(k \cdot \log (S))$ with $S=\mid$ allowed $\left(c_{k}, x_{c h}, \tau^{\prime}\left[x_{c h}\right]\right) \mid$. The worst-case time complexity for one execution of the loop body is then $\mathrm{O}\left(d+\left(k-f_{\min }\right)+k \cdot \log (S)\right)=\mathrm{O}(d+k \cdot \log (S))$. Let us assume that $M$ is the number of sequences of valid tuples that contain no allowed tuple, and for each tuple $\tau^{\prime \prime}$ belonging to such a sequence $\tau^{\prime \prime}\left[\operatorname{scp}\left(c_{k}\right) \cap s c p\left(c_{i}\right)\right]=\tau\left[\operatorname{scp}\left(c_{k}\right) \cap \operatorname{scp}\left(c_{i}\right)\right]$. Then $M$ bounds the number of iterations of the while loop in seekPWsupport. Therefore the worst time complexity of seekPWsupport is $\mathrm{O}(M(d+k \cdot \log (S)))$.

The cost of isPWconsistent+ is $\mathrm{O}(e \cdot M(d+k \cdot \log (S)))$, since in the worst case seekPWsupport is called once for each of the at most $e$ intersecting constraints. The 
maximum number of iterations for the while loop in revise $P W+$ is $N$, where $N$ is the number of sequences of valid tuples in $c_{i}$ containing no allowed tuple. The cost of one call to seekSupport-va is $\mathrm{O}(d+k \cdot \log (S))$ [23]. Therefore, for $d$ values the complexity of revisePW+ is $\mathrm{O}(d \cdot N(e \cdot M(d+k \cdot \log (S))+(d+k \cdot \log (S))))=\mathrm{O}(d \cdot e \cdot N \cdot M(d+$ $k . \log (S))) \cdot \square$

Note that $M$ and $N$ are at most $t_{c_{k}}+1$ and $t_{c_{i}}+1$ respectively, since in the worst case there is a sequence of valid tuples in between every pair of consecutive allowed tuples in a constraint's relation.

The complexity given by Proposition 1 concerns one call to revise $P W+$ for one constraint. If revise $P W+$ is embedded within an AC3-like algorithm (as maxRPWC1 is) to achieve the propagation of all constraints in the problem then the worst-case time complexity of maxRPWC+ will be $\left.\mathrm{O}\left(e^{2} \cdot k \cdot d^{2} \cdot N \cdot M(d+k \cdot \log (S))\right)\right)$ since there are $e$ constraints and each one is enqueued $d k$ times in the worst case (i.e., once for each value deletion from a variable in its scope). Assuming the implementation of described by Lecoutre and Szymanek [23], the space complexity of maxRPWC + is $\mathrm{O}(e . k . \mid$ allowed $(c, x, a) \mid+e . k . d)$, where $|\operatorname{allowed}(c, x, a)|$ is the maximum size of any constraint's relation and $e k d$ is the space required for the Last structure.

Regarding the pruning power of maxRPWC+, it is easy to show that the local consistency it achieves is strictly stronger than GAC (the arguments in the discussion on the pruning power of restricted maxRPWC are directly applicable). Also, the local consistency achieved by maxRPWC+ is incomparable to maxRPWC. This is because a maxRPWC algorithm may achieve stronger pruning than maxRPWC+ due to the detection of PW-support loss in addition to maxRPWC-support loss. On the other hand, the fast check for lack of PW-support enables maxRPWC+ to prune extra values compared to maxRPWC.

Proposition 2 maxRPWC+ achieves a local consistency that is incomparable to maxRPWC.

Proof: For an example where maxRPWC+ achieves more pruning than a maxRPWC algorithm consider Example 1. For the opposite consider a problem with 0-1 domains that includes two constraints $c_{1}$ and $c_{2}$ with $\operatorname{scp}\left(c_{1}\right)=\left\{x_{1}, x_{2}, x_{3}\right\}$ and $\operatorname{scp}\left(c_{2}\right)=$ $\left\{x_{2}, x_{3}, x_{4}\right\}$ having the allowed tuples $\{(0,0,0),(1,0,1),(1,1,0)\}$ and $\{(0,0,0)$, $(0,1,1),(1,0,1)\}$ respectively. Now assume that value 0 is deleted from $D\left(x_{4}\right)$ which means that tuple $(0,0,0)$ of $c_{2}$ will be invalidated. maxRPWC + will revise all other variables involved in $c_{2}$ and only check for maxRPWC-support loss. Both values for $x_{2}$ and $x_{3}$ have maxRPWC-supports on $c_{2}$ so no deletion will be made. On the other hand, a maxRPWC algorithm will also check for PW-support loss by looking at constraint $c_{1}$. It will discover that value 0 of $x_{1}$ is no longer maxRPWC (its GAC-support has no PW-support on $c_{2}$ ) and will therefore delete it.

\subsection{A lighter version of maxRPWC+}

Although maxRPWC+ removes many redundancies that are inherent to generic algorithms through the exploitation of the Last data structure, it suffers from an important drawback: the overhead required for the restoration of Last after a failed instantiation. One way around this problem is to use Last as a residue. That is, as a list of supports 
that have been most recently discovered but are not maintained/restored during search. The resulting algorithm does not remove all the redundancies that maxRPWC+ does, but it is much cheaper to apply during search. This is similar to the relation between the optimal AC algorithm AC2001/3.1 [6] and the residue-based AC $3^{r m}$ [21], as well as, the optimal maxRPC algorithm maxRPC 3 and its corresponding residue-based version $\operatorname{maxRPC} 3^{r m}[2]$.

The residue-based version of maxRPWC + , which hereafter is called maxRPWC $+r$, is an algorithm that exploits backtrack-stable data structures inspired from $\mathrm{AC} 3^{r m}$ and maxRPC $3^{r m}$ ( $r m$ stands for multidirectional residues). The Last structure is not maintained incrementally as by maxRPWC+, but it is only used to store residues. As explained, a residue is a support which has been located and stored during the execution of the procedure that proves that a given tuple is maxRPWC. The algorithm stores the most recently discovered support, but does not guarantee that any lexicographically smaller value is not a maxRPWC-support. Consequently, when we search for a new maxRPWC-support in a table, we always start from scratch. Last need not be restored after a failure; it can remain unchanged, hence a minimal overhead on the management of data.

To obtain the residue-based maxRP $W C+r$ algorithm, we need to make the following simple modifications. In Function 2 we omit line 8, since Last $_{c_{k}, x_{k}, \tau\left[x_{k}\right]}$ may not be the lexicographically smallest tuple in $c_{k}$ and thus, we cannot locate the $\max _{-} \tau^{\prime}$ tuple. Subsequently, in Function $3 \max _{-} \tau^{\prime}$ is set to setNextTuple $\left(c_{i}, \tau, c_{k}, \perp\right.$ ) (namely the search for a PW-support in $c_{k}$ starts from scratch). Additionally, the fast check for lack of PW-support, handled in checkPWtuple (line 2) is not feasible. Lines 1-3 of Function 3 are replaced with the following two:

1: $\max _{-} \tau^{\prime} \leftarrow \operatorname{setNextTuple}\left(c_{i}, \tau, c_{k}, \perp\right)$;

2: if $\max _{-} \tau^{\prime}=\top$ then return $\top$;

Regarding the time complexity of maxRPWC $+r$, the cost of calling Function revise $P W+$ once is the same as in maxRPWC+. However, if we consider that repeated calls may be required due to the effects of constraint propagation then the complexity of maxRPWC+r for the whole problem is $\left.\mathrm{O}\left(e^{2} \cdot k \cdot d^{3} \cdot N \cdot M(d+k \cdot \log (S))\right)\right)$.

Finally, regarding the pruning power of maxRPWC $+r$ it is easy to see that this algorithm achieves a local consistency that is stronger than GAC (again the arguments in the discussion on the pruning power of restricted maxRPWC are directly applicable). Also, it achieves a local consistency weaker than maxRPWC + since the two algorithms are essentially the same, minus the fast check for lack of PW-support.

\section{Extending STR to a higher-order consistency}

Algorithms based on STR, especially STR2 and STR3, have been shown to be the most efficient GAC algorithms for table constraints, along with the MDD approach of [9]. The idea of STR algorithms is to dynamically maintain the tables of supports while enforcing GAC, based on an optimization of simple tabular reduction (STR), a technique proposed by J. Ullmann [34]. 


\subsection{The HOSTR* algorithm}

In this section we present ways to extend STR algorithms in order to achieve more pruning than GAC. From now on we call these algorithms $\operatorname{HOSTR}$, which is derived from higher-order STR. The ' *' stands for a particular STR algorithm (i.e., when extending STR2 we name the algorithm HOSTR2).

Algorithm 5 presents the main framework for HOSTR by extending the basic STR algorithm to achieve a stronger consistency. We choose to present an extension of STR as opposed to STR2 and STR3 because of STR's simplicity. STR2 and STR3 can be extended in a very similar way.

We now present the data structures used by STR and HOSTR.

- $\operatorname{rel}[c]$ : is the set of allowed tuples associated with a positive table constraint $c$. This set is represented by an array of tuples indexed from 1 to $t_{c}$ which denotes the size of the table (i.e., the number of allowed tuples).

- position $[c]$ : is an array of size $t_{c}$ that provides indirect access to the tuples of $c$. At any given time the values in position $[c]$ are a permutation of $\left\{1,2, \ldots, t_{c}\right\}$. The $i^{\text {th }}$ tuple of $c$ is $\operatorname{rel}[c][$ position $[c][i]]$. The use of this data structures enables restoration of deleted tuples in constant time.

- currentLimit $[c]$ : is the position of the last current tuple in $\operatorname{rel}[c]$. The current table of $c$ is composed of exactly currentLimit $[c]$ tuples. The values in position $[c]$ at indices ranging from 1 to currentLimit $[c]$ are positions of the current tuples of $c$.

- $p w V a l u e s[x]$ : is a set for each variable $x$, that contains all values in $D(x)$ which are proved to have a PW-support when HOSTR is applied on a constraint $c$. STR uses a similar data structure to store the values that are GAC-supported.

- $X_{e v t}$ is a set of variables. After HOSTR has finished processing a constraint $c$, $X_{\text {evt }}$ will contain each variable $x \in \operatorname{scp}(c)$ s.t. at least one value was removed from $D(x)$.

Propagation in STR based algorithms can be implemented by means of a queue that handles constraints. Once a constraint is removed from the queue, STR iterates over the valid tuples in the constraint until currentLimit $[c]$ is reached. STR removes any tuple that has become invalid through the deletion of one of its values (just like the while loop in Algorithm 5). Importantly, when a tuple is removed it still remains in rel $[c]$; its index is swapped in position $[c]$ with the index pointed by currentLimit $[c]$ (i.e., the index of the last valid tuple of $\operatorname{rel}[c]$ ) in constant time. Additionally, the only information that is restored upon backtracking is currentLimit $[c]$ instead of the removed tuples. Thus, after finishing the while loop iteration, only valid and allowed tuples are kept in tables. Any value that are no longer supported, are deleted (the for loop in Algorithm 5).

HOSTR is identical to STR, except for the extra PW-check it applies when a tuple is verified as valid. To be precise, if an allowed tuple $\tau$ is proved valid by function isValid then HOSTR checks if it is also PW-consistent. This is done by calling the isPWConsistent-STR function (line 7). 
We omit the detailed description of functions isValid and removeTuple, which can be found in [18]. Briefly, isValid takes a tuple $\tau$ and returns true iff none of the values in $\tau$ has been removed from the domain of the corresponding variable. removeTuple again takes a tuple $\tau$ and removes it in constant time by replacing position $[c][i]$, where $i$ is the position of $\tau$ in $\operatorname{rel}[c]$, with position [c] [currentLimit $[c]]$ (namely by swapping indexes and not tuples) and then decrementing currentLimit $[c]$ by one. As a result this procedure violates the lexicographic ordering of the remaining tuples in position $[c]$.

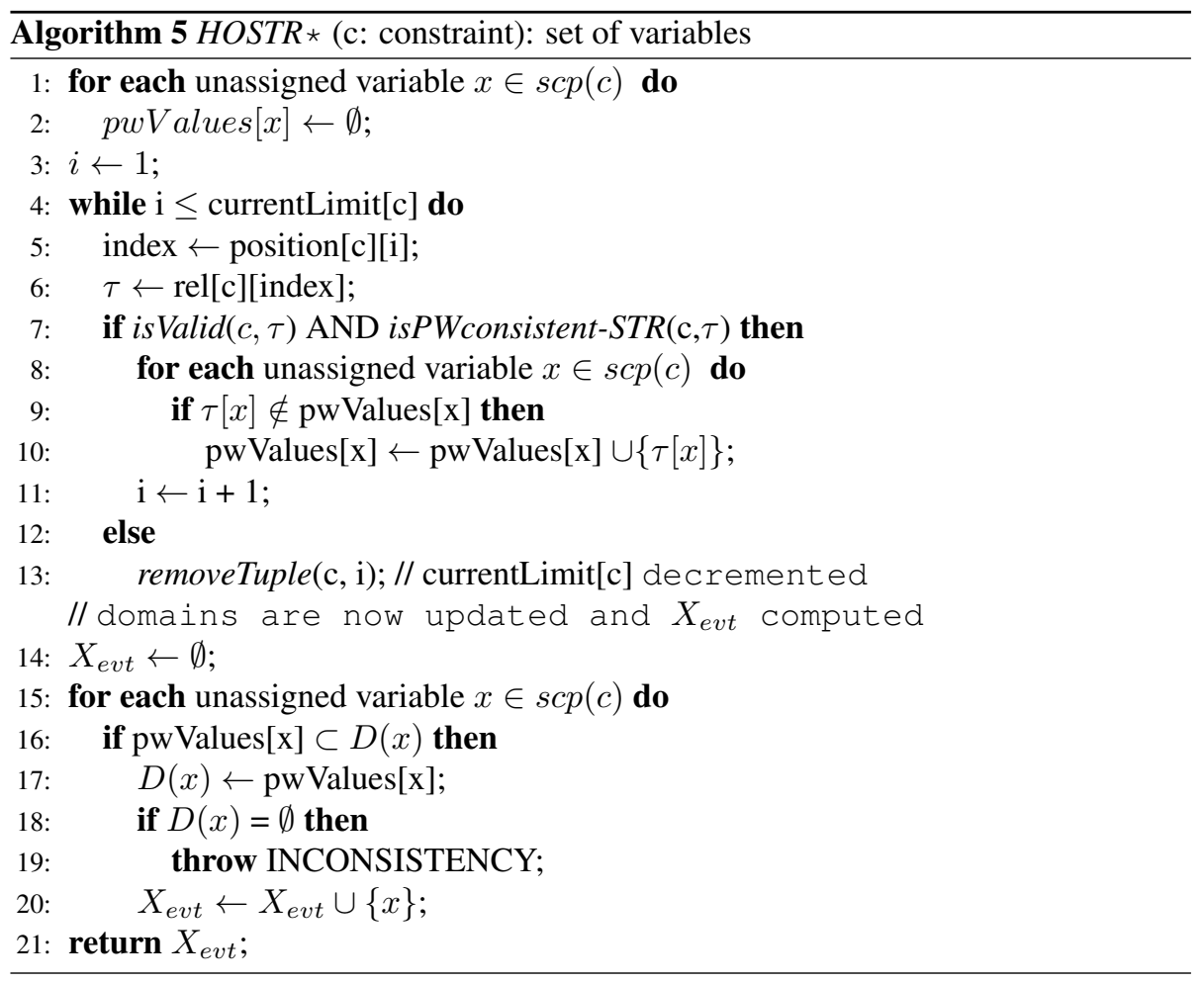

Once a tuple $\tau$ of constraint $c$ has been verified as valid, Function isPWconsistent$S T R$ of HOSTR is called. This function iterates over each constraint $c_{k}$ that intersects with $c$ on at least two variables and searches for a PW-support for $\tau$. If $\tau$ has no PWsupport on some $c_{k}$ then it will be removed in line 13 of Algorithm 5 .

Once the traversal of the valid tuples has terminated, HOSTR (and STR) updates the $X_{e v t}$ set to include any variable that belongs to $s c p(c)$ and has had its domain reduced (lines 14-21). Thereafter, all constraints that involve at least one variable in $X_{\text {evt }}$ will be added to the propagation queue.

Concerning the implementation of isPWconsistent-STR, there are several options, with each one resulting in a different variant of HOSTR. The following variants differ in the way search for a PW-support is implemented.

1. Linear: For each constraint $c_{k}$ isPWconsistent-STR ${ }^{l}$ iterates in a linear fashion over each $\tau^{\prime}$ that currently belongs to $\operatorname{rel}\left[c_{k}\right]$ to locate a valid tuple such that 
$\tau^{\prime}\left[\operatorname{scp}\left(c_{k}\right) \cap s c p(c)\right]=\tau\left[s c p\left(c_{k}\right) \cap s c p(c)\right]$, until currentLimit $\left[c_{k}\right]$ is reached. This version does not require the use of any additional data structures.

2. Binary: For each constraint $c_{k}$ isPWconsistent-STR ${ }^{b}$ locates a $\tau^{\prime}$ by applying a binary search on the initial $\mathrm{rel}\left[c_{k}\right]$. Then it checks its validity and whether it satisfies the condition $\tau^{\prime}\left[s c p\left(c_{k}\right) \cap s c p(c)\right]=\tau\left[s c p\left(c_{k}\right) \cap s c p(c)\right]$. A requirement of this version is that the original table is stored. Note that binary search on the current tables as they are stored by STR is not possible since the lexicographic ordering of the tuples is violated.

3. STR/maxRPWC+ Hybrid: In addition to the data structures of STR, this version keeps the allowed $(c, x, a)$ lists of maxRPWC+ (see Section 3). Then, following maxRPWC+, for each constraint $c_{k}$ isPWconsistent-STR ${ }^{h}$ visits the lists of valid and allowed tuples in an alternating fashion, using binary search, to locate a valid tuple $\tau^{\prime}$ such that $\tau^{\prime}\left[s c p\left(c_{k}\right) \cap \operatorname{scp}(c)\right]=\tau\left[s c p\left(c_{k}\right) \cap s c p(c)\right]$.

Hereafter, we denote by $\operatorname{HOSTR}^{l}$ (respectively $\operatorname{HOSTR}^{b}, \operatorname{HOSTR}^{h}$ ) the HOSTR algorithm that utilizes the function isPWconsistent-STR ${ }^{l}$ (respectively isPWconsistent$S T R^{b}$, isPWconsistent-STR ${ }^{h}$ ).

In practice, the STR/maxRPWC+ hybrid approach (i.e., $\mathrm{HOSTR}^{h}$ ) is by far the most efficient among the above methods. isPWconsistent-STR ${ }^{h}$ which implements this method closely follows the operation of maxRPWC+ when looking for a PW-support, minus the usage of the Last structure. This is displayed in Function 6. For each $c_{k}$ isPWconsistent-STR ${ }^{h}$ calls function setNextTuple to find the lexicographically smallest valid tuple in $c_{k}$, such that $\tau^{\prime}\left[s c p\left(c_{k}\right) \cap s c p(c)\right]=\tau\left[s c p\left(c_{k}\right) \cap s c p(c)\right]$. The search in $c_{k}$ (line 2) starts from scratch (i.e., $\perp$ ), since HOSTR does not store information about recently found maxRPWC-supports. If no such tuple exists, setNextTuple returns $T$, and the search terminates since no PW-support for $\tau$ exists in $c_{k}$. Else, we check if $\tau^{\prime}$ is an allowed tuple using binary search as explained in Function 3.

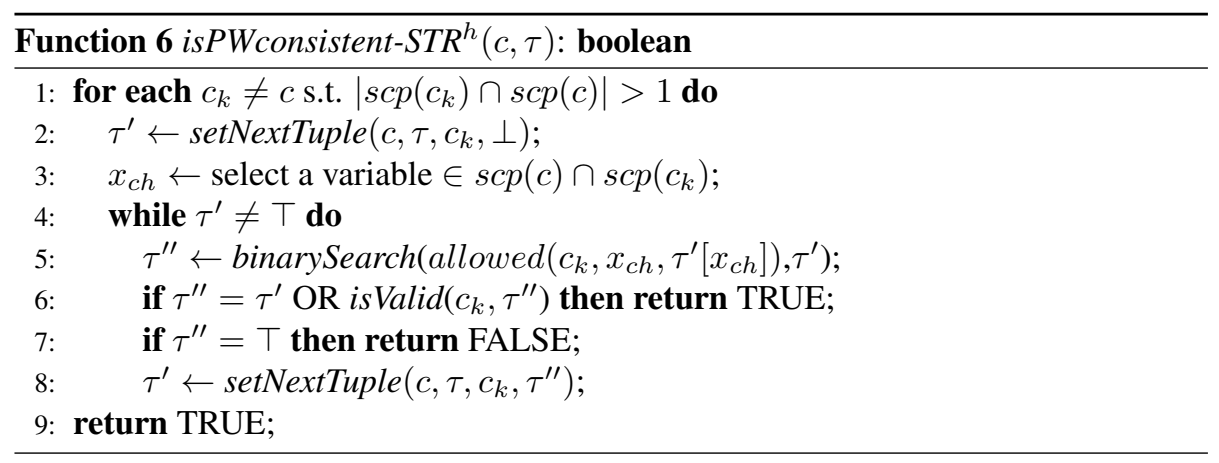

\subsection{Theoretical Results}

For a given constraint $c$, the worst-case time complexity of STR is $\mathrm{O}\left(k^{\prime} d+k t^{\prime}\right)$, where $k^{\prime}$ denotes the number of uninstantiated variables in $\operatorname{scp}(c)$ and $t^{\prime}$ denotes the size 
of the current table of $c$ [18]. The worst-case space complexity of STR is $\mathrm{O}(e k t)$. We now give the worst-case time complexity of HOSTR when implemented using the STR/maxRPWC+ Hybrid approach.

Proposition 3 The worst-case time complexity of HOSTR for the processing of one constraint is $\mathrm{O}\left(k^{\prime} \cdot d+k \cdot t^{\prime}(e \cdot M(d+k \cdot \log (S)))\right)$.

Proof: HOSTR is identical to STR with the addition of the call to isPWconsistent-STR ${ }^{h}$ each time a valid tuple is verified. The time complexity of Function isPWconsistent$S T R^{h}$ is $\mathrm{O}(e \cdot M(d+k \cdot \log (S)))$ (see the proof of Proposition 1 for details). Therefore, the worst-case time complexity of HOSTR is $\mathrm{O}\left(k^{\prime} \cdot d+k \cdot t^{\prime}(e \cdot M(d+k \cdot \log (S)))\right)$.

If HOSTR is embedded within an AC-3 like algorithm to propagate all constraints then the time complexity will be $\mathrm{O}\left(e \cdot d \cdot k \cdot\left(k^{\prime} \cdot d+k \cdot t^{\prime}(e \cdot M(d+k \cdot \log (S)))\right)\right)$ since there are $e$ constraints and each one is enqueued $d k$ times in the worst case (i.e., once for each value deletion from a variable in its scope). The space complexity of HOSTR is $\mathrm{O}(e(k t+k$. allowed $(c, x, a) \mid))$ since in addition to the original tables we need the allowed $(c, x, a)$ lists.

Now we prove that HOSTR, implemented in any of the above ways, achieves a local consistency that is incomparable to that achieved by maxRPWC+ and to maxRPWC, and is weaker than FPWC.

Proposition 4 The local consistency achieved by HOSTR is incomparable to that achieved by maxRPWC+.

Proof: First, consider the problem in Example 1. Since $\max _{-} \tau^{\prime}$ is lexicographically greater than the PW-support of $\tau$ on $c_{2}$ (let us assume that this is the only PW-support for $\tau$ ), this tuple must be PW inconsistent. This means that when HOSTR processes $c_{2}$ it will delete this tuple. If, however, this does not cause any value deletions then it will not be propagated any further and $c_{1}$ will not be processed. Hence, HOSTR will not be able to delete 0 from $D\left(x_{1}\right)$. As explained in Example 1, maxRPWC+ will be able to make this deletion.

To show that HOSTR can delete values that maxRPWC+ cannot delete, consider the problem depicted in Figure 2. There are five variables $x_{1}, \ldots, x_{5}$ with $\{0,1\}$ domains and one variable $\left(x_{6}\right)$ with domain 0 . There are three table constraints with their allowed tuples shown in Figure 2. Value 0 of $x_{1}$ has tuple $(0,0,0)$ as GAC-support in $\mathrm{rel}\left[c_{1}\right]$. This tuple has the PW-support $(0,0,0,0)$ in $r e l\left[c_{2}\right]$, and therefore if maxRPWC+ is applied it will not delete it (as it will not delete any other value). Now assume that HOSTR processes $c_{2}$ first. Tuple $(0,0,0,0)$ does not have a PW-support in $c_{3}$, and therefore it will be removed. When $c_{1}$ is processed, HOSTR will determine that tuple $(0,0,0)$ has no PW-support in $c_{2}$, since $(0,0,0,0)$ has been deleted, and will therefore be removed. As a result, value 0 of $x_{1}$ will loose its only GAC-support in $c_{1}$ and will be deleted.

The extra pruning achieved by HOSTR compared to maxRPWC+ in the above example is a direct consequence of the fact that HOSTR, like STR, removes tuples from constraint relations. Now note that if constraint $c_{1}$ is processed first in the example then no value deletion will be made. This is because when $c_{1}$ is processed tuple $(0,0,0)$ in $\mathrm{rel}\left[c_{1}\right]$ will have the PW-support $(0,0,0,0)$ in $\mathrm{rel}\left[c_{2}\right]$ and therefore value 0 of $x_{1}$ will not be deleted. When $c_{2}$ is later processed, tuple $(0,0,0,0)$ will indeed be removed but 
no value from any of the variables in $\operatorname{scp}\left(c_{2}\right)$ will be deleted. This means that $X_{\text {evt }}$ will be empty and as a result no constraint will be added to the propagation queue. Therefore, $c_{1}$ will not be processed again. Hence, the pruning power of HOSTR cannot be characterized precisely because it depends on the ordering of the propagation queue.

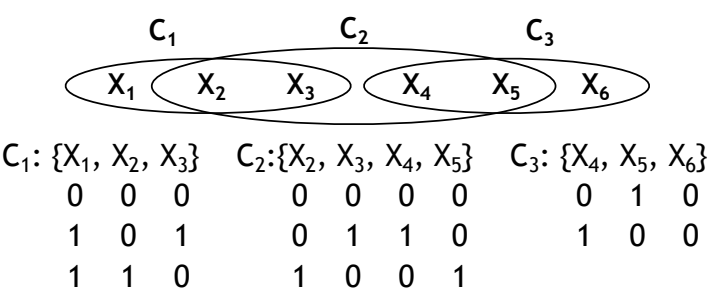

Figure 2: HOSTR vs. maxRPWC+.

Proposition 5 The local consistency achieved by HOSTR is incomparable to maxRPWC.

Proof: First, consider the example of Figure 2. If maxRPWC is applied on this problem it will achieve no pruning. But as explained in the proof of Proposition 4, HOSTR will delete value 0 of $x_{1}$ if $c_{2}$ is processed before $c_{1}$. Therefore, HOSTR can achieve stronger pruning than a maxRPWC algorithm.

Now consider the example in the proof of Proposition 2. After the deletion of value 0 from $D\left(x_{4}\right)$, HOSTR will add $x_{4}$ to $X_{\text {evt }}$ and enqueue $c_{2}$. When $c_{2}$ is then processed no value deletion will be made and therefore propagation will stop. On the other hand, as explained, a maxRPWC algorithm will delete value 0 from $D\left(x_{1}\right)$.

Proposition 6 HOSTR achieves a local consistency that is strictly weaker than PWC+ GAC.

Proof: We first show that any deletion made by HOSTR will also be made by an algorithm that applies FPWC. HOSTR will delete a tuple $\tau \in \operatorname{rel}[c]$ if $\tau$ is invalid or if Function isPWconsistent-STR ${ }^{h}$ cannot find a PW-support for $\tau$ on some constraint $c^{\prime}$. By definition, applying PWC deletes any value that is invalid or not PWC. Hence, it will also delete $\tau$. Correspondingly, HOSTR will delete a value $a \in D(x)$ if it does not participate in any valid and PW consistent tuple on some constraint $c$ that involves $x$. Considering an algorithm that applies FPWC, the PWC phase will delete all invalid and PW inconsistent tuples from $\mathrm{rel}[c]$. Hence, the application of GAC that follows will delete $a$.

Finally, consider the example in Figure 2. As explained, if $c_{1}$ is processed before $c_{2}$, HOSTR will achieve no pruning. In contrast, and independent of the order in which constraints are processed, the application of PWC will remove tuple $(0,0,0,0)$ from $\mathrm{rel}\left[c_{2}\right]$ and because of this tuple $(0,0,0)$ will be removed from $\mathrm{rel}\left[c_{1}\right]$. Then when GAC is applied value 0 will be removed from $D\left(x_{1}\right)$ because it will have no support in $c_{1}$. Hence, FPWC is strictly stronger than HOSTR. 


\subsection{A stronger version of HOSTR}

Motivated by the inability to precisely characterize the pruning power of HOSTR due to its dependence on the propagation order, we propose a simple modification to HOSTR which achieves a stronger consistency property that can be precisely characterized. This algorithm, which we call full HOSTR (fHOSTR) differs from HOSTR in the following: If after traversing the tuples of a constraint $c$, at least one tuple has been removed then all variables that belong to $\operatorname{scp}(c)$ are added to $X_{\text {evt }}$. This means that all constraints that involve any of these variables will be then added to the propagation queue. Recall that HOSTR adds a variable to $X_{e v t}$ only if a value has been deleted from the domain of this variable.

It is easy to see that in the example in Figure 2 fHOSTR will make the same value and tuple deletions as a FPWC algorithm. Generalizing this, we now prove that fHOSTR achieves the level of consistency of FPWC.

Proposition 7 Algorithm fHOSTR achieves FPWC.

Proof: We show that any deletion made by applying FPWC will also be made by fHOSTR. Consider any value $a$ that is removed from a domain after FPWC is applied. This is because all supports for this value on some constraint $c$ have been deleted. These tuples were deleted because they are not valid or not PWC. In the former case, since $f H O S T R$ fully includes the operation of STR, it will delete any invalid tuple when processing constraint $c$ and therefore will also delete value $a$. In the latter case, consider the deletion of any tuple $\tau$ because it is not PWC. This means that all of $\tau$ 's $\mathrm{PW}$-supports on some constraint $c^{\prime}$ have been deleted. When processing $c^{\prime}$, once the last PW-support of $\tau$ is deleted, fHOSTR will enqueues all constraints that intersect with $c^{\prime}$, including $c$. Then when $c$ is processed, fHOSTR will not be able to find a PW-support for $\tau$ on $c^{\prime}$ and will thus delete it. Hence, all support of $a$ will be deleted and as a result $a$ will be deleted.

To complete the theoretical analysis of the algorithm's pruning power, we now show that $\mathrm{EHOSTR}$ achieves a local consistency that is strictly stronger than that achieved by maxRPWC+.

Proposition 8 The local consistency achieved by fHOSTR is strictly stronger than that achieved by maxRPWC+.

Proof: First we show that any deletion made by maxRPWC+ will be also made by fHOSTR. Consider a value $a$ that is removed from $D(x)$ after maxRPWC+ is applied. This is because either:

1. $a$ is not GAC,

2. no GAC-support of $a$ on a constraint $c$ has a PW-support on some constraint $c_{k}$,

3. the deletion is triggered by the "fast check for lack of PW-support".

In the first case, the STR step of fHOSTR will obviously discover that $a$ is not GAC. In the second case, the lack of PW-support for a tuple $\tau$ that includes $a$ on some constraint $c_{k}$ means that no potential PW-support for $\tau$ is valid. When fHOSTR processes tuple $\tau$ 
of $c$ it will try to extend it to a PW-support in all intersecting constraints. Hence, it will consider $c_{k}$ and recognize that $\tau$ has no valid PW-support in $c_{k}$. The same argument holds for all the GAC-supports of $a$ in $c$.

Finally, if $a$ is deleted by the "fast check for lack of PW-support" then the following must hold for each PW-consistent GAC-support $\tau$ for $a$ in some constraint $c$. Any PW-support $\tau^{\prime}$ of $\tau$ in some $c_{k}$ is lexicographically smaller than Last $_{c_{k}, x_{i}, b}$, where $x_{i} \in \operatorname{scp}\left(c_{k}\right)$ is a variable that belongs to the intersection of $c$ and $c_{k}$ and $b$ is its value in $\tau^{\prime}$. maxRPWC+ must have moved the pointer Last $_{c_{k}, x_{i}, b}$ to some tuple $\tau^{\prime \prime}$ beyond the PW-supports of $\tau$ because when trying to find a PW consistent GAC-support for $b$ in $c_{k}$, all tuples lexicographically smaller than $\tau^{\prime \prime}$, including all the PW-supports of $\tau$, were determined as PW inconsistent. Now when fHOSTR processes $c_{k}$ it will determine that all the PW-supports of $\tau$ are not themselves PW consistent and will thus delete them. Therefore, all constraints that involve variables in $\operatorname{scp}\left(c_{k}\right)$, including $c$, will be enqueued. When $c$ is later processed, no PW-support for $\tau$ (or any of $a$ 's GAC-supports in general) will be found, and thus $a$ will be deleted.

For an example where fHOSTR achieves stronger pruning than maxRPWC + consider the second example in the proof of Proposition 4.

Algorithm fHOSTR achieves a stronger local consistency than maxRPWC+ and HOSTR but has a serious drawback: Its time complexity, when embedded within an AC3 like algorithm to propagate all constraints, is $\mathrm{O}\left(e^{2} \cdot t \cdot\left(k^{\prime} \cdot d+k \cdot t^{\prime}(e \cdot M(d+k \cdot \log (S)))\right)\right)$ since there are $e$ constraints and each constraint $c$ is enqueued $\mathrm{O}(e t)$ times in the worst case (i.e., once for each tuple deletion from a constraint intersecting $c$ ). This complexity is prohibitive for large table constraints.

Figure 3 summarizes the relationships between the algorithms discussed throughout this paper with respect to their pruning power. Note that by GAC, maxRPWC, and FPWC we mean any algorithm that achieves these local consistency properties.

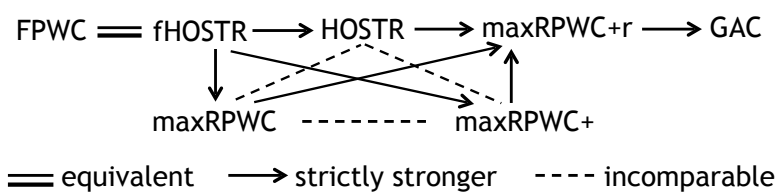

Figure 3: Summary of the relationships between algorithms.

\section{Experiments}

We ran experiments on benchmark non-binary problems with table constraints from the CSP Solver Competition ${ }^{2}$. The arities of the constraints in these problems range from 3 to 18. We tried the following classes: forced random problems, random problems, positive table constraints, BDD, Dubois, and Aim. These classes represent a large spectrum of instances with positive table constraints that are very commonly used for

\footnotetext{
${ }^{2}$ http://www.cril.univ-artois.fr/CPAI08/
} 
the evaluation of GAC algorithms and additionally, non-trivial intersections exist between their constraints. The first two classes only include constraints of arity 3 , while the others include constraints of large arity (up to 18). Note that there exist classes of problems with table constraints where maxRPWC and similar methods do not offer any advantage compared to GAC because of the structure of the constraints. For example, on crossword puzzles constraints intersect on at most one variable. Our algorithms cannot achieve extra filtering compared to GAC in such problems and thus we have not included them in this study.

In more detail, the 150 tried instances belong to classes that have the following attributes:

- The first two series Random-fcd and Random involve 20 variables and 60 ternary relations of almost 3,000 tuples each.

- The series $B D D$ involves 21 Boolean variables and 133 constraints generated from binary decision diagrams of arity 18 that include 58,000 tuples.

- The two series Positive table-8 and Positive table-10 contain instances that involve 20 variables. Each instance of the series Positive table-8 (resp.,Positive table-10) involves domains containing 5 (resp. 10) values and 18 (resp. 5) constraints of arity 8 (resp. 10). The constraint tables contain about 78,000 and 10,000 tuples, respectively.

- The Dubois class contains instances involving 80 Boolean variables and quaternary constraints.

- The Aim-100 and Aim-200 series involve 100 and 200 Boolean variables respectively, with constraints of small arities (mainly ternary and a few binary).

The algorithms were implemented within a CP solver, written in Java, and tested on an Intel Core i5 of 2.40GHz processor and 4GB RAM. A CPU time limit of 6 hours was set for all algorithms and all instances. Search used the dom/ddeg heuristic for variable ordering and lexicographical value ordering. We have chosen dom/ddeg [5] as opposed to the generally more efficient $d o m / w d e g$ [8] because the decisions made by the latter are influenced by the ordering of the propagation queue making it harder to objectively compare the pruning efficiency of the algorithms [3]. Having said this, experiments with $\mathrm{dom} / \mathrm{wdeg}$ did not give significantly different results compared to dom/ddeg as far as the relative efficiency of the algorithms is concerned.

We present results from our baseline implementations of STR2 and maxRPWC1, upon which the new algorithms were built, compared to the proposed algorithms, HOSTR $2^{h}$, maxRPWC+ and maxRPWC $+r$. For maxRPWC1 we used its residual version in order to avoid the maintenance of the LastGAC structure during search. This resulted in faster run times. We also include results from our later designed algorithm eSTR $2^{w}$. We do not include results from $\mathrm{fHOSTR} 2^{h}$, since its restricted version, that approximates FPWC, is always more efficient. The differences between fHOSTR $2^{h}$ and HOSTR $2^{h}$ in favour of the latter range from being marginal to 3 times faster. As explained in Section 4.3 this is due to the high cost of applying the full algorithm. 
Finally, we also compare our algorithms to the state-of-the-art solver Abscon ${ }^{3}$. Specifically, we compare against Abscon's implementations of the GAC algorithms GAC-Va and STR2, denoted by GAC-va_abs and STR2_abs respectively. Although Abscon's implementation is much more optimized than ours, these results help to put our contributions in context of modern CP solvers and point out classes of problems where our methods are very effective.

\subsection{Preprocessing}

Table 1 shows the mean CPU times (in milliseconds) obtained by the tested algorithms on each problem class for the initialization (i) and the preprocessing (p) phase. During initialization, the data structures of a specific algorithm are initialized, while preprocessing includes one run of a specific filtering algorithm before the search commences. Therefore, initialization time is the time required for each algorithm to construct all its structures, while preprocessing time is the time for a stand alone use of a specific algorithm.

Our implementation for STR2 is the fastest algorithm in the initialization phase in all tested classes, while both Abscon's GAC algorithms are faster in the preprocessing phase. It is notable that STR2_abs and GAC-va_abs require at least one order of magnitude more time to construct their structures compared to our STR2, hinting that they utilize intricate data structures that are later exploited throughout search (e.g., for fast restoration upon backtracking).

Table 1: Mean CPU times of the initialization (i) and the preprocessing (p) phase in milliseconds from various problem classes.

\begin{tabular}{|c|c|c|c|c|c|c|c|c|c|}
\hline Problem Class & & STR2 & STR2_abs & GAC-va_abs & maxRPWC1 & HOSTR2 $^{h}$ & maxRPWC+r & maxRPWC+ & eSTR2 $^{w}$ \\
\hline \multirow[t]{2}{*}{ Random- $f c d$} & $\mathrm{i}$ & 31 & 646 & 678 & 115 & 345 & 202 & 263 & 689 \\
\hline & $\mathrm{p}$ & 111 & 15 & 0 & 696 & 399 & 154 & 250 & 97 \\
\hline \multirow[t]{2}{*}{ Random } & i & 19 & 644 & 677 & 10 & 249 & 249 & 272 & 616 \\
\hline & $\mathrm{p}$ & 87 & 17 & o & 738 & 291 & 186 & 247 & 85 \\
\hline \multirow[t]{2}{*}{ Positive table- 8} & i & 83 & 2,654 & 3,280 & 5 & 1,305 & 1,510 & 1,628 & 76,062 \\
\hline & $\mathrm{p}$ & 271 & 82 & 0 & 2,891 & 36,621 & 343 & 359 & 952 \\
\hline \multirow[t]{2}{*}{ Positive table-10 } & i & 2 & 601 & 653 & $\mathbf{0}$ & 236 & 263 & 304 & 12,214 \\
\hline & $\mathrm{p}$ & 47 & 34 & 2 & $4,997,817$ & 363,000 & 620,210 & 772,193 & 20 \\
\hline \multirow[t]{2}{*}{$B D D$} & $\mathrm{i}$ & 237 & 697 & 755 & 123 & 10,017 & 8,530 & 8,334 & mem \\
\hline & $\mathrm{p}$ & 1,415 & 622 & 6 & 477,497 & 2,218 & 6,159 & 16,875 & mem \\
\hline \multirow[t]{2}{*}{ Dubois } & $\mathrm{i}$ & 10 & 423 & 415 & 12 & 13 & 10 & 12 & 10 \\
\hline & $\mathrm{p}$ & $\mathbf{0}$ & 2 & 0 & 5 & $\mathbf{0}$ & 2 & 2 & 2 \\
\hline \multirow[t]{2}{*}{ Aim-100 } & i & 108 & 431 & 435 & 126 & 244 & 111 & 195 & 213 \\
\hline & $\mathrm{p}$ & 2 & 3 & 2 & 160 & 19 & 19 & 38 & 12 \\
\hline \multirow[t]{2}{*}{ Aim-200 } & i & 397 & 513 & 492 & 303 & 465 & 270 & 280 & 319 \\
\hline & $\mathrm{p}$ & 4 & 1 & 4 & 174 & 30 & 53 & 97 & 11 \\
\hline
\end{tabular}

Algorithms for strong local consistencies, as expected, spend extra time to record the intersections of the constraints during the initialization, while they are more expensive when they are applied stand-alone (i.e., during preprocessing). Interestingly, maxRPWC1 has low initialization times, since it does not use the allowed data structure that most proposed strong local consistency algorithms use. On the contrary,

\footnotetext{
${ }^{3}$ http://www.cril.univ-artois.fr/ lecoutre/software
} 
maxRPWC1 is by far inferior compared to all other algorithms during preprocessing. Particularly, on Positive table- 8 and Positive table-10 it is more than two orders of magnitude worse than all proposed algorithms.

Regarding the initialization times of the three proposed strong consistency algorithms, maxRPWC+r appears to be the fastest. HOSTR ${ }^{h}$ needs more time for the initialization since it uses the structures of both STR2 and maxRPWC+. Concerning the preprocessing, results are more varied since there are classes where maxRPWC+r dominates HOSTR ${ }^{h}$ and vice versa. This is due to the different approaches of STRlike and GAC-va-like algorithms, as the former iterate over tuples and the latter over values and tuples, thus it is not clear which one is preferable for stand-alone use. On the other hand, it is clear that maxRPWC+r, being lighter, is faster than maxRPWC+.

eSTR2 ${ }^{w}$ is the fastest algorithm during preprocessing among the strong algorithms. Particularly, its times are very close to the ones of STR2 due to their close time-complexity. On the contrary, it requires more time for the initialization phase in order to build its structures. This is evident especially on Positive table- 8 and Positive table-10 because the tables are long and constraints intersect on many variables ( 7 and 8 respectively). On $B D D$, where constraints intersect on 16 variables, eSTR $2^{w}$ reveals its main drawback as it exhausts all of the available memory.

Finally, the high preprocessing times for all proposed methods on the Positive table10 class are due to the high memory consumption on these large instances. However, as we show below, preprocessing by these algorithms is able to determine the unsatisfiability of the instances without requiring search. However, on Positive table- 8 both maxRPWC+ and maxRPWC+r are very close to STR2 and two (resp. one) orders of magnitude faster compared to HOSTR2 ${ }^{h}$ (resp. maxRPWC1) preprocessing times.

\subsection{Search}

In Table 2 we present selected representative results from search algorithms that apply the tested filtering algorithms throughout search, while in Table 3 we give the mean and median performance of the search algorithms in each problem class ${ }^{4}$. Note that results from class Aim-200 were obtained using the $\mathrm{dom} / \mathrm{wdeg}$ variable ordering heuristic. This is because our algorithms were unable to solve these problems within the time limit using $\mathrm{dom} / \mathrm{d} d \mathrm{deg}$.

All of the tried algorithms completed all instances within the cutoff limit except for maxRPWC1, which did not solve 13 instances out of 20 from the Positive table- 8 class. Also, eSTR $2^{w}$ solved none of the $B D D$ instances since it exhausted the available memory on each one of them.

Among the three proposed algorithms, maxRPWC $r$ is the most efficient with HOSTR $2^{h}$ being a close second. maxRPWC $+r$ is faster than HOSTR $2^{h}$ on Random, Random-fcd and especially on Positive table-8 (e.g., on the rand-8-20-5-18-800-12 instance it is over 6 times faster), while it is slower on Positive table-10. On the rest of the classes HOSTR $2^{h}$ is better than maxRPWC $+r$, but without considerable differences. maxRPWC $+r$ is also constantly faster compared to maxRPWC+, with the differences being considerable in the Dubois and Aim classes.

\footnotetext{
${ }^{4}$ These results include initialization and preprocessing times.
} 
Comparing our algorithms against maxRPWC1 we observe that they are particularly efficient on instances of large arities. These are the instances of the Positive Table and $B D D$ classes, whose arities vary from 8 to 18 . Both maxRPWC $+r$ and maxRPWC + are notably faster, especially on Positive table- 10 and $B D D$ they are superior by over one order of magnitude. There are also cases (i.e., Positive table-8), where maxRPWC1 was not able to solve the majority of the instances within the cutoff limit.

Table 2: CPU times ( $\mathrm{t}$ ) in secs and nodes (n) from various representative problem instances. A slash (-) means that the instance was not completed within the time limit. mem means that memory was exhausted.

\begin{tabular}{|c|c|c|c|c|c|c|c|c|c|}
\hline Instance & & STR2 & STR2_abs & GAC-va_abs & maxRPWC1 & HOSTR2 ${ }^{h}$ & $\operatorname{maxRPWC}+\mathrm{r}$ & maxRPWC+ & eSTR2 ${ }^{w}$ \\
\hline rand $-3-20-20$ & $\mathrm{t}$ & 242 & 53 & $\mathbf{3 0}$ & 308 & 291 & 237 & 195 & 79 \\
\hline $60-632-f c d-5$ & $\mathrm{n}$ & 160,852 & 156,394 & 156,394 & 66,335 & 66,601 & 66,469 & 66,585 & 86,110 \\
\hline rand $-3-20-20$ & $\mathrm{t}$ & $\begin{array}{l}90 \\
\end{array}$ & 20 & 13 & 174 & 131 & 78 & 82 & 31 \\
\hline $60-632-f c d-7$ & $\mathrm{n}$ & 73,536 & 72,178 & 72,178 & 19,680 & 19,791 & 19,988 & 18,668 & 16,432 \\
\hline rand $-3-20-20$ & $\mathrm{t}$ & 472 & 178 & 91 & 867 & 879 & 567 & 564 & 200 \\
\hline $60-632-5$ & $\mathrm{n}$ & 501,583 & 479,945 & 479,945 & 152,712 & 152,763 & 153,138 & 152,892 & 150,893 \\
\hline rand $-3-20-20$ & $\mathrm{t}$ & 16 & & 4 & 32 & 38 & 18 & 17 & 11 \\
\hline $60-632-14$ & $\mathrm{n}$ & 19,996 & 16,511 & 16,511 & 3,976 & 3,986 & 4,002 & 3,898 & 3,578 \\
\hline rand-8-20-5 & $\mathrm{t}$ & 17 & 8 & 7 & 1,616 & 1,860 & 494 & 754 & 246 \\
\hline $18-800-7$ & $\mathrm{n}$ & 17,257 & 19,341 & 19,341 & 3,424 & 3,444 & 3,447 & 3,430 & 1,001 \\
\hline rand $-8-20-5$ & $t$ & 19 & 12 & 16 & - & 12,625 & 2,823 & 3,654 & 147 \\
\hline $18-800-12$ & $\mathrm{n}$ & 105,521 & 95,895 & 95,895 & - & 28,830 & 28,752 & 28,662 & 15,517 \\
\hline rand $-10-20$ & $\mathrm{t}$ & 0.4 & 0.6 & 6 & 3,811 & 174 & 203 & 208 & 11 \\
\hline $10-5-10000-1$ & $\mathrm{n}$ & 1,110 & 1,111 & 1,111 & 0 & $\mathbf{0}$ & $\mathbf{0}$ & $\mathbf{0}$ & $\mathbf{0}$ \\
\hline rand-10-20 & $\mathrm{t}$ & 0.3 & 0.6 & 2.5 & 6,438 & 1,283 & 1,212 & 1,298 & 10 \\
\hline $10-5-10000-4$ & $\mathrm{n}$ & 1,110 & 1,110 & 1,110 & 0 & 0 & 0 & 0 & $\mathbf{0}$ \\
\hline$b d d-21-133$ & $\mathrm{t}$ & 30 & 11 & 18 & 2.4 & 0.6 & 1.5 & 2 & mem \\
\hline $18-78-6$ & $\mathrm{n}$ & 20,582 & 20,617 & 20,617 & 0 & $\mathbf{0}$ & $\mathbf{0}$ & $\mathbf{0}$ & - \\
\hline bdd-21-133 & $\mathrm{t}$ & 39 & 11 & 21 & 1,714 & 1.2 & 11.6 & 16.8 & mem \\
\hline $18-78-11$ & $\mathrm{n}$ & 19,364 & 19,670 & 19,670 & 21 & 21 & 21 & 21 & \\
\hline \multirow[t]{2}{*}{ dubois-21 } & $\mathrm{t}$ & 110 & 69 & 49 & 56 & 40 & 53 & 314 & 50 \\
\hline & $\mathrm{n}$ & $58 \mathrm{M}$ & $56 \mathrm{M}$ & $56 \mathrm{M}$ & 19M & $23 \mathrm{M}$ & $23 \mathrm{M}$ & $23 \mathrm{M}$ & $23 \mathrm{M}$ \\
\hline \multirow[t]{2}{*}{ dubois-26 } & $\mathrm{t}$ & 4,044 & 2,898 & 1,799 & 3,427 & 1,463 & 1,830 & 12,174 & 2,183 \\
\hline & $\mathrm{n}$ & $1,823 \mathrm{M}$ & $2,147 \mathrm{M}$ & $2,147 \mathrm{M}$ & $808 \mathrm{M}$ & $744 M$ & $744 M$ & $744 M$ & $744 M$ \\
\hline aim-100 & $t$ & 6,423 & 336 & 169 & 0.4 & 0.15 & 0.18 & 0.25 & 0.2 \\
\hline 1-6-sat-2 & $\mathrm{n}$ & $29 \mathrm{M}$ & $59 \mathrm{M}$ & $59 \mathrm{M}$ & 100 & 100 & 100 & 100 & 100 \\
\hline aim-100 & $\mathrm{t}$ & 14,862 & 185 & 99 & 3,378 & 5,211 & 3,766 & 15,617 & 3,334 \\
\hline 2-0-sat-4 & $\mathrm{n}$ & $1,729 \mathrm{M}$ & $20 M$ & $20 \mathrm{M}$ & $51 \mathrm{M}$ & $149 \mathrm{M}$ & $149 \mathrm{M}$ & $149 \mathrm{M}$ & $149 \mathrm{M}$ \\
\hline aim-200 & $\mathrm{t}$ & 57 & & 3.6 & 0.5 & 0.4 & 0.5 & 0.6 & 0.9 \\
\hline 2-0-sat-1 & $\mathrm{n}$ & $2 \mathrm{M}$ & 575,943 & 575,943 & 257 & 2,210 & 2,210 & 1,782 & 9,847 \\
\hline aim-200 & $\mathrm{t}$ & 0.7 & 2.2 & 1.3 & 2.7 & 2.5 & 5.9 & 1.3 & 1.8 \\
\hline 2-0-unsat-1 & $\mathrm{n}$ & 23,715 & 97,558 & 97,558 & 16,091 & 46,893 & 34,671 & 34,671 & 41,177 \\
\hline
\end{tabular}

eSTR2 ${ }^{w}$ is superior to the proposed algorithms by a factor of 3 on Random and Random-fcd while on Aim it displays a close performance. Also, it is one order of magnitude faster on Positive table-8 and Positive table-10. These results are not surprising given than eSTR ${ }^{w}$ is a more recent algorithm based on a different concept than the algorithms presented here. However, as noted before, eSTR $2^{w}$ suffers from a serious drawback: its high memory consumption in the presence of large constraint intersections. Hence, on the $B D D$ class it fails to even construct its data structures. This is because in this class constraints intersect on 16 variables and along with the big size of the tables the use of eSTR2 becomes prohibitive. In such kind of problems the need for algorithms with lighter data structures is mandatory and demonstrates the practical advantages of our approaches. 
Now comparing Abscon to our algorithms, both STR2_abs and GAC-va_abs are much faster on the Random and Positive table classes. Counterwise, our algorithms typically outperform Abscon on $B D D$ and Dubois, even by orders of magnitude in some cases (e.g., HOSTR2 ${ }^{h}$ on bdd-21-133-18-78-6). This is the class where none of the GAC algorithms could detect unsatisfiability through preprocessing unlike strong consistency algorithms. In comparison with our implementation of STR2, the differences are even larger in favor of HOSTR $2^{h}$ and maxRPWC $+r$ on the same classes, albeit they are significantly outperformed on Positive table instances. We believe that our approaches will become more efficient and competitive as soon as they are integrated in an optimized implementation, such as that of Abscon. Albeit, even with the current implementation, they display important gains in many cases (i.e., in $B D D$, Dubois, and Aim instances).

Table 3: Mean CPU times $(t)$ in secs, mean node visits $(n)$, median CPU times $\left(t_{m}\right)$ and median node visits $\left(n_{m}\right)$ from various problem classes.

\begin{tabular}{|c|c|c|c|c|c|c|c|c|c|}
\hline Class & & STR2 & STR2_abs & GAC-va_abs & maxRPWC1 & HOSTR2 $^{h}$ & maxRPWC+r & maxRPWC+ & eSTR2 $^{w}$ \\
\hline Random & $t$ & 152 & 43 & 28 & 310 & 305 & 176 & 171 & 71 \\
\hline \multirow[t]{3}{*}{$-f c d$} & $n$ & 132,492 & 156,326 & 156,326 & 49,009 & 49,317 & 49,420 & 48,608 & 45,272 \\
\hline & $t_{m}$ & 131 & 28 & 18 & 214 & 256 & 126 & 117 & \\
\hline & $n_{m}$ & 105,423 & 95,995 & 95,995 & 31,399 & 31,503 & 31,610 & 30,727 & 27,222 \\
\hline \multirow[t]{4}{*}{ Random } & $t$ & 54 & 63 & 39 & 461 & 501 & 298 & 288 & 116 \\
\hline & $n$ & 211,456 & 215,763 & 215,763 & 79,928 & 80,172 & 80,316 & 78,802 & $\mathbf{7 3 , 5 3 3}$ \\
\hline & $t_{m}$ & & 36 & 18 & 269 & 318 & 157 & 155 & 75 \\
\hline & $n_{m}$ & 102,327 & 95,417 & 95,417 & 41,243 & 41,301 & 41,322 & 41,107 & 40,715 \\
\hline Positive & $t$ & 15 & 9 & 11 & - & 5,002 & 1,168 & 1,576 & 134 \\
\hline \multirow[t]{3}{*}{ table- 8} & $n$ & 52,313 & 46,478 & 46,478 & - & 10,087 & 9,787 & 10,039 & 4,818 \\
\hline & $t_{m}$ & & 10 & 11 & 2,416 & 4,194 & 1,089 & 1,470 & 133 \\
\hline & $n_{m}$ & 45,880 & 39,923 & 39,923 & 7,721 & 8,134 & 7,979 & 8,112 & 3,274 \\
\hline Positive & $t$ & 0.3 & 0.8 & 3.5 & 4,998 & 363 & 620 & 773 & 12 \\
\hline \multirow[t]{3}{*}{ table-10 } & $n$ & 1,110 & 1,110 & 1,110 & 0 & $\mathbf{0}$ & 0 & 0 & $\mathbf{0}$ \\
\hline & $t_{m}$ & 0.4 & 0.8 & 2 & 5,723 & 367 & 1,240 & 1,381 & 10 \\
\hline & $n_{m}$ & 1,110 & 1,110 & 1,110 & 0 & 0 & 0 & 0 & $\mathbf{0}$ \\
\hline \multirow[t]{4}{*}{$B D D$} & $t$ & 30 & 10 & 19 & 924 & 3 & 18 & 24 & mem \\
\hline & $n$ & 19,139 & 19,012 & 19,012 & 11 & 11 & 11 & 11 & \\
\hline & $t_{m}$ & & 11 & 20 & 634 & 1 & 6 & 9 & mem \\
\hline & $n_{m}$ & 20,956 & 20,587 & 20,587 & 11 & 11 & 11 & 11 & - \\
\hline \multirow[t]{4}{*}{ Dubois } & $t$ & 2,026 & 1,432 & 906 & 1,413 & 807 & 1,005 & 6,751 & 1,084 \\
\hline & $n$ & $1,008 \mathrm{M}$ & $1,070 \mathrm{M}$ & $1,070 \mathrm{M}$ & $419 \mathrm{M}$ & $401 M$ & $401 \mathrm{M}$ & $401 M$ & $401 \mathrm{M}$ \\
\hline & $t_{m}$ & 673 & 481 & 318 & 563 & 264 & 369 & 2,372 & 371 \\
\hline & $n_{m}$ & $359 \mathrm{M}$ & $373 \mathrm{M}$ & $373 \mathrm{M}$ & $156 \mathrm{M}$ & $141 \mathrm{M}$ & $141 \mathrm{M}$ & $141 \mathrm{M}$ & $141 \mathrm{M}$ \\
\hline \multirow[t]{4}{*}{ Aim-100 } & $t$ & 6,390 & 136 & 70 & 748 & 1,019 & 863 & 3,899 & 674 \\
\hline & $n$ & $643 \mathrm{M}$ & $18 \mathrm{M}$ & $18 \mathrm{M}$ & $10 \mathrm{M}$ & $34 \mathrm{M}$ & $34 \mathrm{M}$ & $34 \mathrm{M}$ & $32 \mathrm{M}$ \\
\hline & $t_{m}$ & 1,435 & 97 & 50 & 0.8 & 22 & 14 & 94 & 0.2 \\
\hline & $n_{m}$ & $103 \mathrm{M}$ & $13 \mathrm{M}$ & $13 \mathrm{M}$ & 1,131 & 875,979 & 875,985 & 875,985 & 1,522 \\
\hline \multirow[t]{4}{*}{ Aim-200 } & $t$ & 13 & 11 & 5 & 3 & 4 & $\mathbf{3}$ & 15 & 4 \\
\hline & $n$ & 479,073 & 846,357 & 846,357 & 14,937 & 97,529 & 104,748 & 88,541 & 75,209 \\
\hline & $t_{m}$ & 2.7 & 2.5 & 1.5 & 0.6 & 0.6 & 0.4 & 1.3 & 0.6 \\
\hline & $n_{m}$ & 159,591 & 120,897 & 120,897 & 937 & 2,887 & 2,887 & 3,236 & 4,276 \\
\hline
\end{tabular}

The results are more varied on the Aim classes where the winning algorithm is not clear. On most instances there are (often huge) differences in favor of our methods compared to Abscon. However, there are cases where Abscon visits considerably fewer nodes than our algorithms, resulting in much better run times. This is contrary to what is expected since a stronger local consistency should typically result in a smaller tree size. However, the interaction with the variable ordering heuristic is sometimes 
unpredictable, given also the different implementations of Abscon and our solver. For example, if we look at the results from aim-100-2-0-sat-4 we can see a huge difference in node visits between Abscon and our implementation of STR2. This is because, despite both applying GAC, the two solvers follow quite different search paths. Consequently, the comparison of our methods on Aim is more meaningful against our own implementation of STR2. In this case, our methods typically outperform STR2 by very large margins.

Looking at the mean and median performances in Table 3, maxRPWC $+r$ (and HOSTR $2^{h}$ ) is faster than STR2 in the BDD, Dubois and Aim classes with the differences being considerable in the two Aim classes. On the other hand, STR2 dominates in the Random and Positive table classes, with the differences being very significant in the latter. Median CPU times follow the average CPU times in the majority of the classes, but they are more representative on Aim where results are diverse, since there are instances solved very quickly and instances solved in thousands of seconds.

Regarding the pruning power of the strong local consistency algorithms, which is to some extent reflected on node visits, it is worth noticing that the differences between the strong consistency methods are negligible in the majority of the classes. The Aim classes are exceptions as maxRPWC1 visits significantly less nodes compared to our algorithms, and this results in competitive CPU times. Importantly, the very small differences in node visits between maxRPWC1 and our algorithms on problems with constraints of large arity (Positive table and $B D D$ ) mean that the very large differences in run times can only be explained by the algorithmic optimizations that we propose in this paper.

\subsubsection{Pairwise comparisons}

CPU times from all tested instances comparing maxRPWC $+r$ to HOSTR ${ }^{h}$, maxRPWC1, STR2, and STR2_abs are presented in Figures 4, 5, 6, 8, respectively, in a logarithmic scale. Different signs display instances from different problem classes and are calculated by CPU time ratios of the compared algorithms. Points placed above the diagonal correspond to instances that were solved faster by maxRPWC+r.

In Figure 4 most instances are gathered around the diagonal indicating closely matched performance between maxRPWC $+r$ and HOSTR $2^{h}$. maxRPWC $+r$ is superior to HOSTR $2^{h}$, as detailed above, on Positive table- 8 and inferior on BDD and Aim-100, but without significant differences.

Comparing maxRPWC $+r$ to maxRPWC1 in Figure 5, we can see the benefits of our approach: Only a few instancesfrom $B D D$ and Aim are below the diagonal indicating that maxRPWC $+r$ is clearly superior to maxRPWC1. Also, there are many instances where maxRPWC1 thrashes while maxRPWC+r does not.

Looking at Figure 6 we see a more varied picture. Although on most instances maxRPWC $+r$ and STR2 are closely matched, there are numerous instances where one of the two methods thrashes and vice versa. This demonstrates that efficient algorithms for strong local consistencies on table constraints constitute a useful addition to the standard GAC approach, but at the same time further research is required to develop methods that can be more robust than state-of-the-art GAC algorithms such as STR2 on a wider range of problems. 


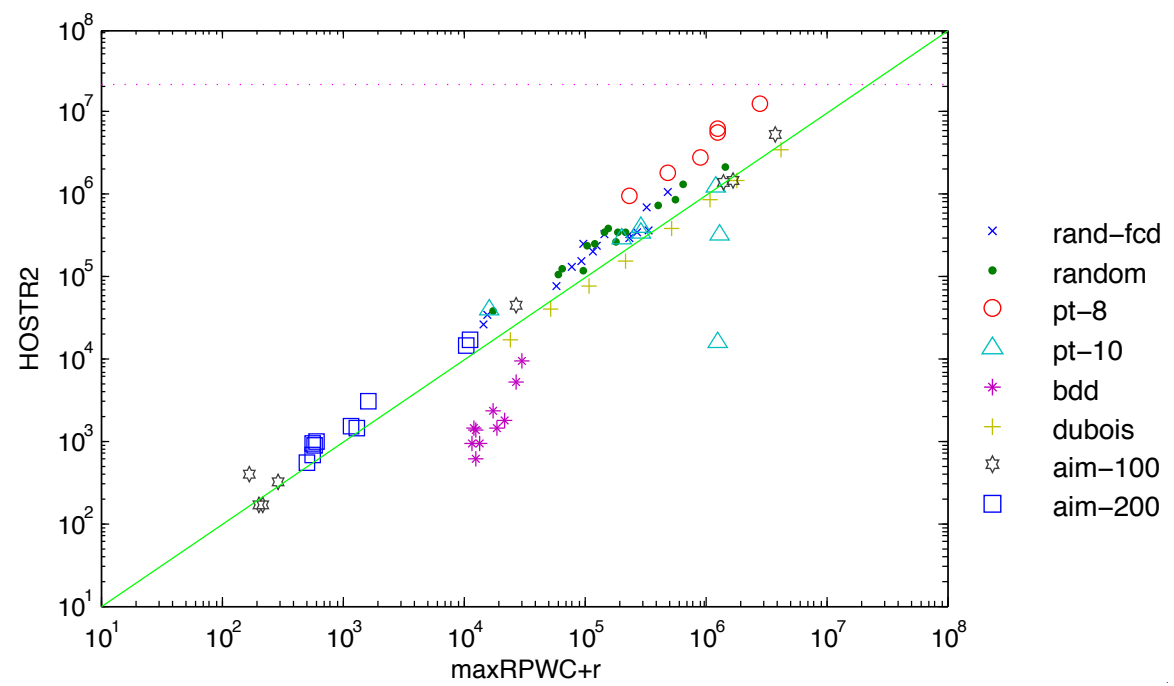

Figure 4: HOSTR2 ${ }^{h}$ vs. maxRPWC+r.

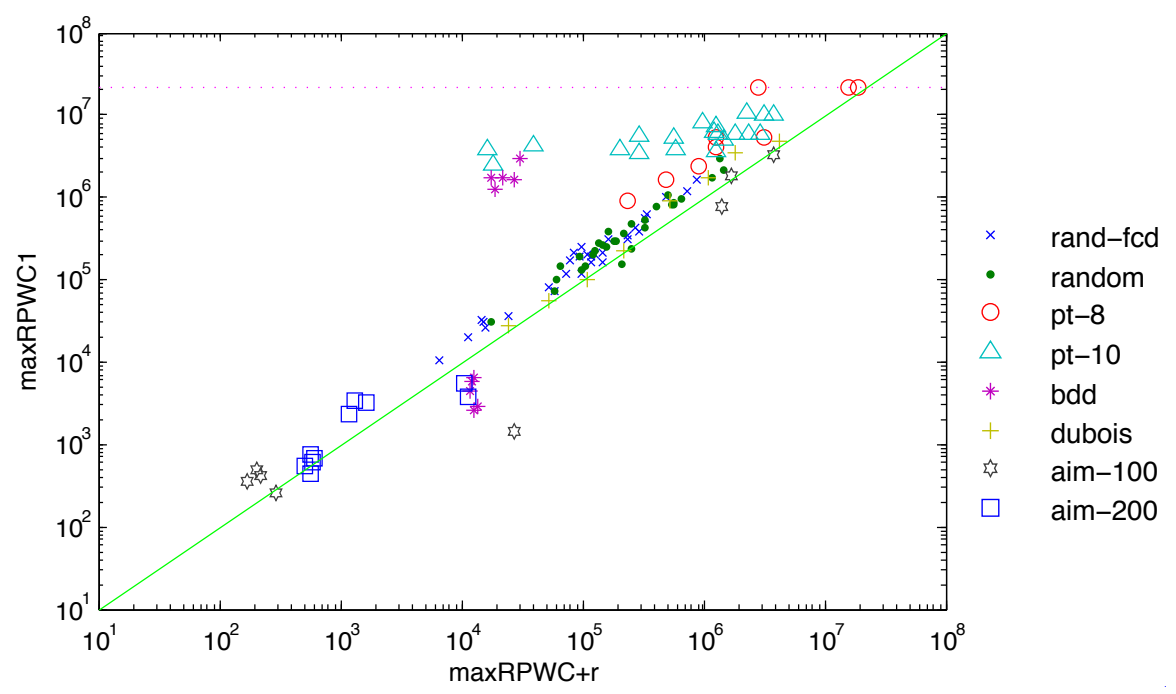

Figure 5: $\operatorname{maxRPWC1}$ vs. $\operatorname{maxRPWC}+r$.

A similar pattern emerges when comparing HOSTR2 ${ }^{h}$ to STR2. This is shown in Figure 7. Specifically, HOSTR $2^{h}$ is superior on instances with constraints of small arity but many constraint intersections, like Dubois and Aim. On the other hand, despite being inferior on many instances from both the Positive table classes, it is faster by an order of magnitude on $B D D$ instances where the intersections between constraints include up to 16 variables. 


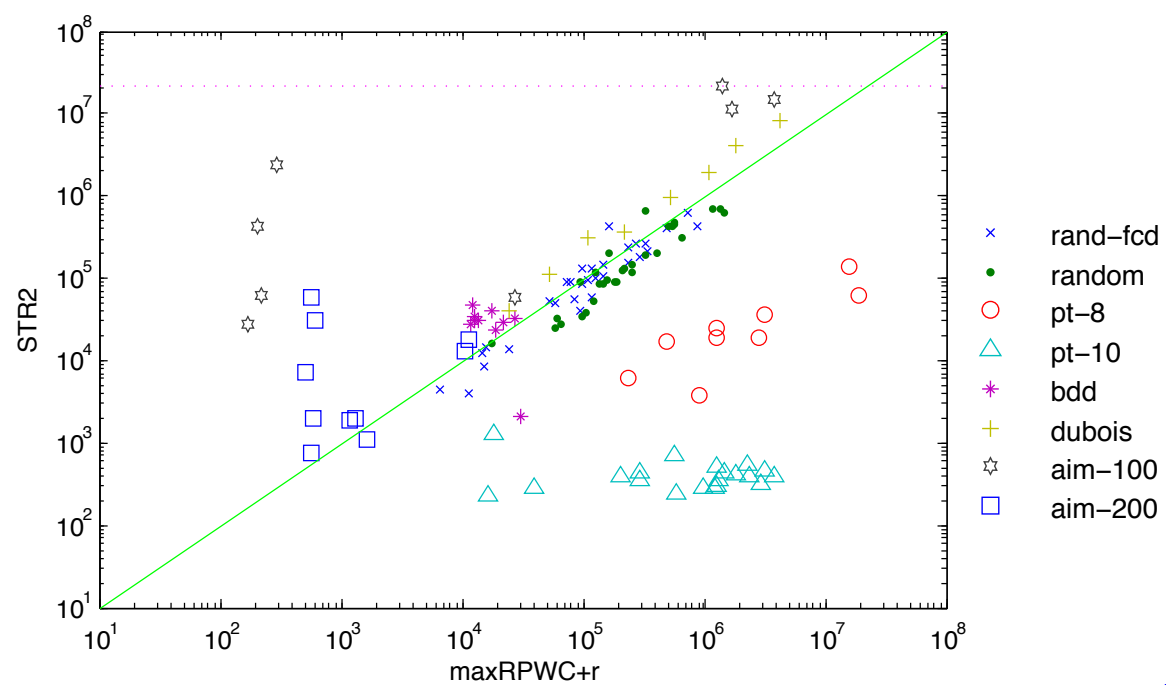

Figure 6: STR2 vs. maxRPWC+r.

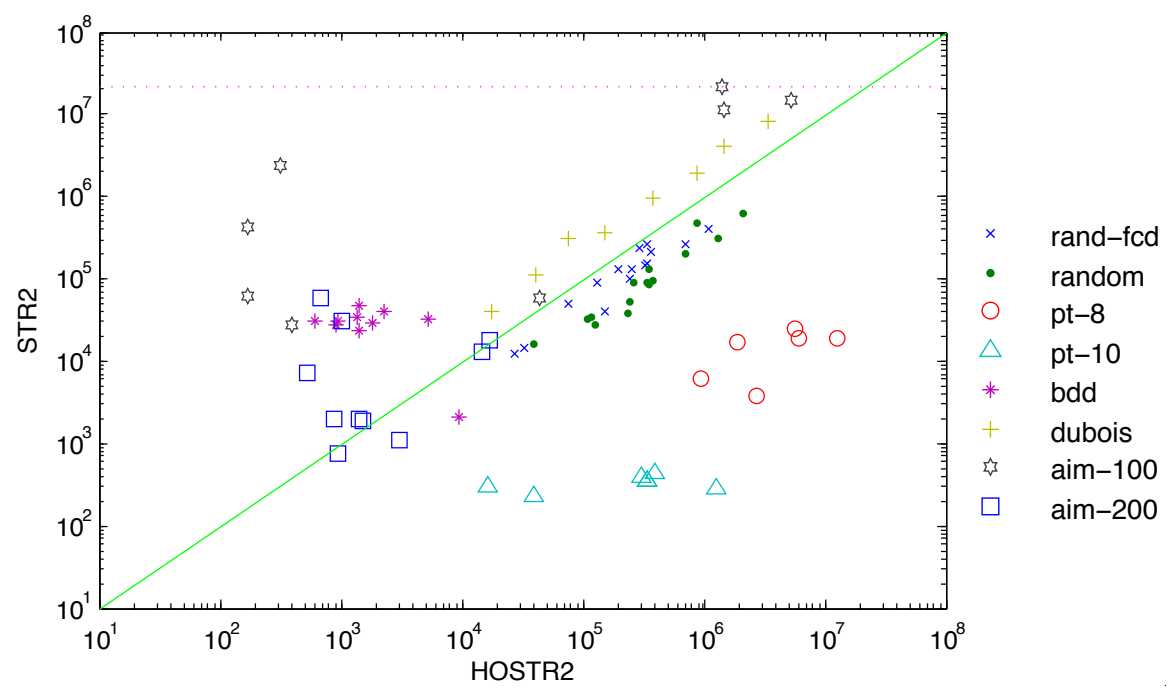

Figure 7: STR2 vs. HOSTR2 ${ }^{h}$.

In Figure 8 we compare STR2_abs to maxRPWC+r. Results from a comparison between GAC-va_abs and maxRPWC+r are very similar and thus we do not present the corresonding figure. Although Figure 8 displays significant differences in favor of STR2 _abs on some instances, there are still quite a few instances where maxRPWC+r is superior, often by large margins. This shows the potential of strong local consistencies on table constraints. As discussed, we believe that integrating an algorithm like maxRPWC $+r$ in Abscon, or some other state-of-the-art solver, would result in much better run time performance. 


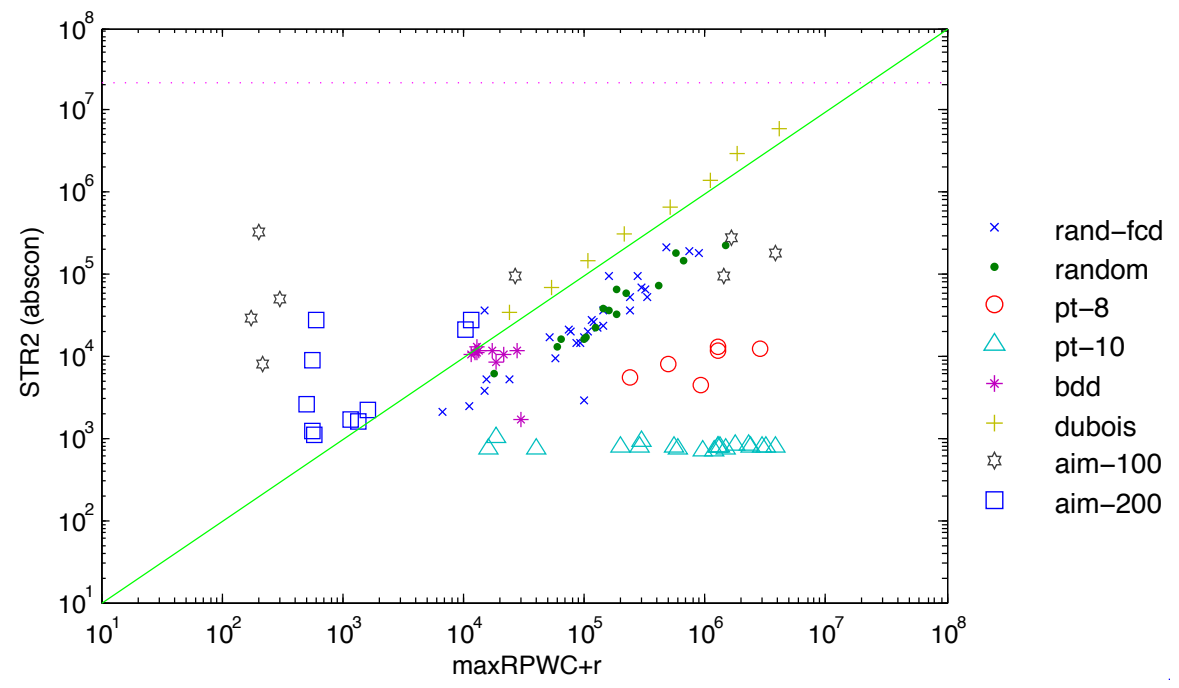

Figure 8: STR2_abs vs. maxRPWC+r.

\subsubsection{Discussion}

We now draw some general conclusions correlating the performance of the proposed algorithms and the characteristics of the tested problems, based on the experimental results.

First, it is evident that the performance of our methods is highly dependant on the presence of non-trivial constraint intersections as their exploitation offers extra pruning compared to GAC algorithms. The absense of non-trivial intersections makes these methods obsolete.

Second, the arity of the constraints, the number of shared variables between constraints, and the size of the tables are important factors when comparing our algorithms to existing maxRPWC algorithms. This is because for large arity constraints the improvements in saving constraint checks and avoiding redundant operations on data structures become more evident. Results from the classes that include constraints of large arity (Positive table, $B D D$ ) confirm this.

However, the above factors do not appear to be decisive when comparing our methods to GAC algorithms. There are problems with small arities as well as others with large arities where our methods are better (resp. worse) than GAC algorithms, i.e., the last (resp. the first) four classes of Table 3. The same holds when considering the size of the tables. Also, as explained, the number of shared variables is a decisive factor when comparing against ESTR because this algorithm may run out of memory when this number is high.

Third, the domain size of the variables seems to be an important factor when comparing our methods to GAC algorithms or eSTR. Our methods are mostly competitive on problems with small domains (BDD, Dubois, Aim). This is because all of our methods include, to some extent, an interleaved exploration of valid and allowed tuples. For problems with large domain sizes the number of valid tuples can be quite high, at least 
near the top of the search tree when only a few value deletions have been made. This can slow down the interleaved search for supports significantly. eSTR does not have this problem because it employs quite different reasoning.

\section{Related Work}

GAC algorithms for table constraints have attracted considerable interest dating back to GAC-Schema [4]. This generic method can be instantiated to either a method that searches the lists of allowed tuples for supports, or to one that searches the valid tuples. The GAC-va algorithm improves on GAC-Schema by interleaving the exploration of allowed and valid tuples using binary search [23]. The interleaved exploration of allowed and valid tuples is also the main idea in [26]. However, in this case it is implemented through the use of an elaborate data structure (Hologram) introduced by Lhomme [25]. Recent algorithms are partly based on similar ideas as the Hologram method [29]. That is, they hold information about removed values in the propagation queue and utilize it to speed up support search.

Alternative data structures for storing and handling table constraints have been also introduced [11]. In that paper, a Trie structure was the most efficient among the ones tested. Katsirelos and Walsh used a compact representation for allowed and disallowed tuples which can be constructed from a decision tree that represents the original tuples [17].

Simple Tabular Reduction (STR) [34] and its variants, STR2 [18] and STR3 [22], constitute an alternative and efficient approach to enforcing GAC based on the dynamic maintenance of the support tables. Finally, multi-valued decision diagrams have been used to store and process table constraints [9].

Experimental results show that the most competitive approaches are the ones based on STR and the MDD approach. The algorithm of [23], which maxRPWC+ extends, is very competitive with the Trie approach, outperforms the Hologram method and has the advantage of easier implementation and lack of complex data structures over all other methods. Albeit, it is clearly slower than the best methods on most problems.

With respect to strong local consistencies, there is considerable older work on relation filtering consistencies. Such methods take advantage of the intersections between constraints in order to identify and remove inconsistent tuples or to add new constraints to the problem (e.g., [35, 13]). Quite recently, strong domain filtering consistencies have received attention [7, 32]. Moreover, efficient ways to apply relational consistencies were proposed and new consistencies of this type were introduced for binary constraints $[19,14,38]$ and non-binary constraints [16, 37].

The works of Woodward et. al. concern the application of various relational consistencies on (mainly) table constraints through the exploitation of a problem's dual encoding [16, 37]. Experimental results show that very high local consistencies (higher than FPWC) can pay off when they are applied through efficient algorithms. However, the proposed algorithms were not compared to state-of-the-art GAC methods such as STR2. Interestingly, the so called index-tree data structure was proposed to efficiently locate all tuples in a constraint's relation that are consistent with a tuple of another intersecting constraint [16]. This is a contribution that is orthogonal to ours and it would 
be interesting to use this data structure in the context of our algorithms.

The idea of avoiding checks for PW-support loss that algorithms maxRPWC+ and maxRPWC $+r$ employ is inspired by a similar idea concerning maxRPC, a related local consistency property for binary constraints $[36,1,2]$. Although the restricted versions of maxRPC algorithms (called light maxRPC [36]) achieve only an approximation of maxRPC, they are much faster compared to full maxRPC algorithms, and they very close in terms of pruning. In addition, the idea of making a lighter use of the Last structure by not maintaining it during search, which is employed by maxRPWC+r, is inspired by the residue-based $\mathrm{AC} 3^{r m}$ [21] and maxRPC $3^{r m}$ algorithms [2].

Furthermore, both EHOSTR and ESTR [24] achieve the same level of consistency, namely GAC+PWC. Their difference lies in the different data structures they utilize which results in a different handling of PW-checks in their respective isPWconsistent function. Whenever a tuple $\tau \in \operatorname{rel}[c]$ is verified as valid, both algorithms iterate over each constraint $c_{k}$ that intersects with $c$ to check if there exists a PW-support for $\tau$ in $\mathrm{rel}\left[c_{k}\right]$. In $\mathrm{eSTR}$, this is done through a look-up in the appropriate counter stored for each intersection between any two constraints. At any time each counter holds the number of valid tuples in $c_{k}$ 's relation table that include a specific combination of values for the set of variables that are common to both $c$ and $c_{k}$. Importantly, this check is done in constant time, while in fHOSTR we need to search between valid and allowed tuples (for the case of function 6). However, experiments in this paper showed that there are cases where while strong consistency methods outperform GAC, the use of HOSTR or maxRPWC $+r$ is inevitable (i.e., in problems with large constraint intersections) because eSTR exhausts the memory resources.

Importantly, strong local consistency techniques for table constraints have recently started to find their way into state-of-the-art CP solvers such as Abscon ${ }^{5}$. The latest version of Abscon offers an unpublished algorithm which achieves FPWC and can be regarded as a variant of $\operatorname{eSTR}^{w}$ [24]. This development demonstrates that strong local consistency methods, such as the ones presented in this paper, are compatible with $\mathrm{CP}$ solvers from a software engineering point of view, and can used to extend the solvers' arsenal of techniques for handling table constraints.

Finally, a simple and efficient way to incorporate strong local consistency reasoning into CP solvers, through the use of a reformulation, was very recently proposed [27]. The main idea of the technique, called factor encoding, is to formulate new variables by extracting commonly shared variables from the constraints' scopes and then to reattach them back to the constraints where they come from. The reformulation transforms a given problem into another equivalent one, and by enforcing GAC on the new problem, a quite stronger consistency property is achieved on the original problem. This approach was compared experimentally to the FPWC algorithm of Abscon with favourable results.

\section{Conclusion}

We presented specialized algorithms for table constraints that achieve local consistencies stronger than the standard GAC. These algorithms build on and extend existing

\footnotetext{
${ }^{5} \mathrm{http}: / /$ www.cril.univ-artois.fr/ lecoutre/software.html
} 
algorithms for GAC and maxRWPC and contribute to both directions of domain and relation filtering local consistencies. Experimental results demonstrated the usefulness of the proposed algorithms in the presence of intersecting table constraints, showing that the best among them can be competitive with a state-of-the art GAC algorithm (STR2) and sometimes even with the state-of-the-art CP solver Abscon.

In some cases (e.g., Aim classes) there can be huge differences in the numbers of search tree nodes which are reflected on important CPU time gains, even compared to a highly optimized solver like Abscon. However on other problems the extra processing performed by our methods slows down the solver, sometimes considerably (e.g the Positive table classes).

Our paper binds together recent advances on GAC for table constraints and strong local consistencies contributing to both directions. Specifically, we offer efficient methods for strong filtering in cases of intersecting table constraints, and we make strong consistencies more practical by moving from generic to specialized algorithms.

We believe that the presented work can pave the way for the design and implementation of even more efficient strong consistency methods for table constraints. Also, it can perhaps help initiate a wider study on specialized strong consistency algorithms for specialized (global) constraints.

\section{Acknowledgements}

We owe a great thanks to Christophe Lecoutre for giving us the executable of the Abscon solver. We also thank the anonymous reviewers for helping us improve the quality of this paper.

\section{References}

[1] T. Balafoutis, A. Paparrizou, K. Stergiou, and T. Walsh. Improving the performance of maxRPC. In Proceedings of CP'10, pages 69-83, 2010.

[2] T. Balafoutis, A. Paparrizou, K. Stergiou, and T. Walsh. New algorithms for max restricted path consistency. Constraints, 16(4):372-406, 2011.

[3] T Balafoutis and K. Stergiou. Exploiting constraint weights for revision ordering in arc consistency algorithms. In ECAI-08 Workshop on Modeling and Solving Problems with Constraints, 2008.

[4] C. Bessiere and J.C. Régin. Arc consistency for general constraint networks: preliminary results. In Proceedings of IJCAI'97, pages 398-404, 1996.

[5] C. Bessiere and J.C. Régin. MAC and combined heuristics: two reasons to forsake FC (and CBJ?). In Proceedings of CP'96, pages 61-75, Cambridge MA, 1996.

[6] C. Bessiere, J.C. Régin, R. Yap, and Y. Zhang. An optimal coarse-grained arc consistency algorithm. Artificial Intelligence, 165(2):165-185, 2005. 
[7] C. Bessiere, K. Stergiou, and T. Walsh. Domain filtering consistencies for nonbinary constraints. Artificial Intelligence, 172(6-7):800-822, 2008.

[8] F. Boussemart, F. Hemery, C. Lecoutre, and L. Sais. Boosting systematic search by weighting constraints. In Proceedings of ECAI'04, Valencia, Spain, 2004.

[9] K. Cheng and R. Yap. An MDD-based generalized arc consistency algorithm for positive and negative table constraints and some global constraints. Constraints, 15(2):265-304, 2010.

[10] R. Debruyne and C. Bessiere. Domain filtering consistencies. JAIR, 14:205-230, 2001.

[11] I. P. Gent, C. Jefferson, I. Miguel, and Nightingale P. Data structures for generalised arc consistency for extensional constraints. In Proceedings of AAAI'07, pages 191-197, 2007.

[12] P. Janssen, P. Jégou, B. Nouguier, and M.C. Vilarem. A filtering process for general constraint satisfaction problems: Achieving pairwise consistency using an associated binary representation. In Proceedings of IEEE Workshop on Tools for Artificial Intelligence, pages 420-427, 1989.

[13] P. Jégou. On the consistency of general constraint satisfaction problems. In Proceedings of AAAI'93, pages 114-119, 1993.

[14] P. Jégou and C. Terrioux. A new filtering based on decomposition of constraint sub-networks. In Tools with Artificial Intelligence (ICTAI), 2010 22nd IEEE International Conference on, volume 1, pages 263-270, 2010.

[15] U. Junker. Preference-based problem solving for constraint programming. In CSCLP, pages 109-126, 2007.

[16] S. Karakashian, R. Woodward, C. Reeson, B. Choueiry, and C. Bessiere. A first practical algorithm for high levels of relational consistency. In Proceedings of AAAI'10, pages 101-107, 2010.

[17] G. Katsirelos and T. Walsh. A compression algorithm for large arity extensional constraints. In Proceedings of CP'07, pages 379-393. Springer-Verlag, 2007.

[18] C. Lecoutre. STR2: optimized simple tabular reduction for table constraints. Constraints, 16(4):341-371, 2011.

[19] C. Lecoutre, S. Cardon, and J. Vion. Conservative dual consistency. In Proceedings of AAAI'07, pages 237-242, 2007.

[20] C. Lecoutre, S. Cardon, and J. Vion. Second-order consistencies. J. Artif. Int. Res., 40(1):175-219, 2011.

[21] C. Lecoutre and F. Hemery. A study of residual supports in arc cosistency. In Proceedings of IJCAI'07, pages 125-130, 2007. 
[22] C. Lecoutre, C. Likitvivatanavong, and R. H. C. Yap. A path-optimal GAC algorithm for table constraints. In ECAI, pages 510-515, 2012.

[23] C. Lecoutre and R. Szymanek. Generalized arc consistency for positive table constraints. In Proceedings of CP'06, pages 284-298, 2006.

[24] Christophe Lecoutre, Anastasia Paparrizou, and Kostas Stergiou. Extending STR to a higher-order consistency. In Proceedings of AAAI'13, pages 576-582, 2013.

[25] O. Lhomme. Arc-consistency filtering algorithms for logical combinations of constraints. In Proceedings of CPAIOR'04, pages 209-224, 2004.

[26] O. Lhomme and J.C. Régin. A fast arc consistency algorithm for n-ary constraints. In Proceedings of AAAI'05, pages 405-410, 2005.

[27] C. Likitvivatanavong, W. Xia, and R. H. C. Yap. Higher-order consistencies through GAC on factor variables. In Proceedings of CP'14, pages 497-513, 2014.

[28] A. Mackworth. On reading sketch maps. In Proceedings of IJCAI'77, pages 598-606, Cambridge MA, 1977.

[29] J.B. Mairy, P. Hentenryck, and Y. Deville. An optimal filtering algorithm for table constraints. In $C P$, pages 496-511, 2012.

[30] R. Mohr and T. Henderson. Arc and path consistency revisited. Artificial Intelligence, 28:225-233, 1986.

[31] A. Paparrizou and K. Stergiou. An efficient higher-order consistency algorithm for table constraints. In Proceedings of AAAI' 12, pages 535-541, 2012.

[32] K. Stergiou. Strong inverse consistencies for non-binary CSPs. In Proceedings of ICTAI'07, pages 215-222, 2007.

[33] K. Stergiou and T. Walsh. Inverse Consistencies for non-binary constraints. In Proceedings of ECAI'06, pages 153-157, 2006.

[34] J. R. Ullmann. Partition search for non-binary constraint satisfaction. Inf. Sci., 177(18):3639-3678, 2007.

[35] P. van Beek and R. Dechter. On the minimality and global consistency of rowconvex constraint networks. JACM, 42(3):543-561, 1995.

[36] J. Vion and R. Debruyne. Light algorithms for maintaining max-RPC during search. In Proceedings of SARA'09, 2009.

[37] R. J. Woodward, S. Karakashian, B. Y. Choueiry, and C. Bessiere. Solving difficult CSPs with relational neighborhood inverse consistency. In Proceedings of AAAI'11, pages 112-119, 2011.

[38] R. J. Woodward, S. Karakashian, B. Y. Choueiry, and C. Bessiere. Revisiting neighborhood inverse consistency on binary CSPs. In Proceedings of CP'12, pages 688-703, 2012. 